\title{
PERTURBATION ANALYSIS FOR INVESTMENT PORTFOLIOS UNDER PARTIAL INFORMATION WITH EXPERT OPINIONS
}

\author{
J.-P. FOUQUE* ${ }^{*}$ A. PAPANICOLAOU ${ }^{\dagger}$, AND R. SIRCAR ${ }^{\ddagger}$
}

\begin{abstract}
We analyze the Merton portfolio optimization problem when the growth rate is an unobserved Gaussian process whose level is estimated by filtering from observations of the stock price. We use the Kalman filter to track the hidden state(s) of expected returns given the history of asset prices, and then use this filter as input to a portfolio problem with an objective to maximize expected terminal utility. Our results apply for general concave utility functions. We incorporate time-scale separation in the fluctuations of the returns process, and utilize singular and regular perturbation analysis on the associated partial information HJB equation, which leads to an intuitive interpretation of the additional risk caused by uncertainty in expected returns. The results are an extension of the partially-informed investment strategies obtained by the Black-Litterman model, wherein investors' views on upcoming performance are incorporated into the optimization along with any degree of uncertainty that the investor may have in these views.
\end{abstract}

Key words. Filtering, control, Hamilton-Jacobi-Bellman equation, portfolio optimization, partial information, expert opinions.

Subject classifications. 91G20, 60G35, 35Q93, 35C20

1. Introduction. It is well-known that optimal mean-variance portfolio weights are quite different from the weights used in real-life investment. As pointed out in [BL92], unconstrained optimization often results in large short positions among many asset classes, and constrained optimization results in zero weight in many assets and unreasonably large weights in assets with small capitalization. The model of Black and Litterman [BL91] is a practical solution to this problem, wherein investors' views on upcoming performance are incorporated into the optimization along with any degree of uncertainty that the investor may have in these views.

In continuous time or multi-period settings, a natural extension of the Black and Litterman model is to include a separate stochastic process for the level of expected returns. Due to the large amount of data required for accurate estimation of this new process, much of the existing literature considers it to be unobserved, hence making this an investment problem with only partial information. Several papers consider expected returns to be an unobserved Markov process and then use filtering methods (i.e. Kalman filter or Wonham filter) to track the hidden state. Indeed, investment with partial information is studied in [Bre98, Bre06, ESB10, BR05, FPS14, Pap13, Pha09, SH04]. The effect of this added stochasticity is that optimal investment strategies will change from those suggested by the standard Merton problem [Mer69], with the effects of riskaversion characteristics and the investment horizon playing a role in the solution [Bre98, Wac02].

The analysis in [FGW12] combines filtering with so-called expert opinions, a concept which is similar to the Black-Litterman model's consideration of investors' views. The idea is the following: in a dynamic investment problem, the nature of the expert opinions is that they are available sporadically over time, and are useful because they can reduce the variance of the estimator of the returns. During the time intervals between the announcement of expert opinions, the investors use a filter to estimate the posterior distribution of the expected returns. In the model of [FGW12],

\footnotetext{
*Department of Statistics \& Applied Probability, University of California, Santa Barbara, CA 93106-3110, fouque@pstat.ucsb.edu. Work supported by NSF grant DMS-1107468.

†School of Mathematics \& Statistics, The University of Sydney, NSW 2006, Australia alpapani@maths.usyd.edu.au.

‡Department of ORFE, Princeton University, E-Quad, Princeton NJ 08544; sircar@princeton.edu. Work partially supported by NSF grant DMS-1211906.
} 
expert opinions arrive with the jumps of a Poisson process, and it allows for a varying degree of investor confidence by including some randomness in the information provided at each arrival time.

1.1. History \& Motivation. One of the earliest papers in modern portfolio theory is that of Markowitz [Mar52], which used the basic statistics of mean and covariance to quantify risk and to find diversified portfolios. In the seminal work of Merton [Mer69], the dynamic investment problem was formulated in continuous time using stochastic control theory, and has since been referred to as the Merton problem. In its original formulation, the Merton problem seeks to optimize utility of consumption and terminal wealth at some finite time, when asset prices evolve according to geometric Brownian motion, and the investor's utility is typically of HARA or CARA type (i.e. the power or exponential utilities, respectively). In Merton's approach, the problem is solved using the Hamilton-Jacobi-Bellman (HJB) equation of dynamic programming.

In the years since [Mer69], there has been a long line of research that relaxes some of the original assumptions in order to make the model more realistic, for instance by making the expected returns stochastic, making volatility stochastic, allowing for transaction costs, or incorporating the unobservability of the parameters. In particular, stochasticity of expected returns has been used in a number of papers: as a possible way to explain greater long-term allocations in stocks than in bonds [CV99, Wac02]; to study the risk-aversion of investors under greater uncertainty than a standard CAPM [BEW88]; to say something more about the equity premium puzzle [Bre98].

1.2. Literature Review. For general surveys on the optimal investment problem we refer the reader to [Pha09, Sch03] and [Rog13]. The Black-Litterman model was first presented in [BL91, BL92]. The model is compared to the Bayesian approach to traditional portfolio theory in [SWZ10]. Models where stochastic volatility is driven by multiscale observable factors are analyzed in [FPSS11, JS02b, JS02a, FSZ13]. The full information portfolio problem with a mean-reverting stochastic drift has been studied by [Wac02] for the complete market (perfectly correlated) case. The relationship of partial information to the preference for higher allocation ratio given longtime horizons, the effects of risk aversion levels and the equity premium puzzle are studied in [Bre98, CV99].

Portfolio optimization with partial information has been studied in [SH04], with Markov chain switching in [BR05, Pap13], and with the effects of discrete trading in [BUV12]. The effects of model parameters such as time-dependent risk aversion and correlation were studied in [FPS14, KO96, SD14]. In [Car09, Pap13], filtering is applied in the management of commodities portfolios with partial information due to the unobservability of convenience yields. Asymptotics analysis for control problems with Markov processes is described in [YZ13], and limit theorems for (nonlinear) filtering have been addressed in [PRS11] and in [PS14]. The effects on filtering with intermittent insertion of expert opinions is studied in [FGW12].

1.3. Results in this Paper. In this paper, we analyze the Merton problem when the growth rate is an unobserved Gaussian process whose level is estimated by filtering from observations of the stock price. We use the Kalman filter to track the hidden state(s) of expected returns given the history of asset prices, and then use this filter as input to a Merton-type portfolio problem with an objective to maximize expected terminal utility of wealth. Our results apply for general concave utility functions.

We incorporate time-scale separation in the factors driving the returns process, and perform singular and regular perturbation analysis on the associated partial information HJB equation, which leads to an intuitive interpretation of the additional risk caused by uncertainty in expected returns. In particular, a fast mean-reverting factor in the expected returns process captures the 
effects of intraday trading, and a slow mean-reverting factor in expected returns captures a slight lack of confidence in the expert opinions.

Our perturbation analysis is carried out in the dual formulation obtained by a Fenchel-Legendre transform, and is then mapped back to the primal formulation. This is a good approach to the problem because the dual PDE to the nonlinear HJB equation is linear. In the later sections we carry out numerical experiments to illustrate the effects of partial information and time-scale perturbations. The chief contribution of this paper is an intuitive set of results relating the model's parameters to the role of partial information in optimal investment.

The rest of the paper is organized as follows: Section 2 introduces the model; Section 2.1 explains the filter and Section 2.2 presents the small- $\varepsilon$ and small- $\delta$ asymptotics of the Kalman filter in the regime of small time scale parameters $\varepsilon$ and $\delta$. Section 2.3 explains how expert opinions affect uncertainty; Section 3 presents the partial information HJB equation, with Section 3.2 deriving the linearized PDE for the dual function. Section 4 presents the small- $\varepsilon$ (fast factor) asymptotics. Section 5 presents the small- $\delta$ (slow factor) expansions; and Section 6 shows how the partial information value functions compare to those from full information case, and that an information premium is seen qualitatively. Finally, the Appendices contain various proofs, including a novel application of the Hörmander theorem to show existence of smooth solutions to the dual PDE.

2. Model for Stochastic Returns \& Filtering Equations. We consider a market with two investment options: a risk-free bank account and a risky asset whose price is the observed process in the following system:

$$
\begin{array}{rlrl}
\frac{d S_{t}}{S_{t}} & =\left(\theta+Y_{t}+Z_{t}\right) d t+\sigma d W_{t} & & \text { (observed price) } \\
d Y_{t} & =-\frac{1}{\varepsilon} Y_{t} d t+\frac{\beta}{\sqrt{\varepsilon}} d B_{t}^{1} & \text { (hidden) } \\
d Z_{t} & =-\delta Z_{t} d t+\sqrt{\delta} \eta d B_{t}^{2} & \text { (hidden) }
\end{array}
$$

where $W, B^{1}$ and $B^{2}$ are Brownian motions with

$$
\mathbb{E}\left\{d W_{t} d B_{t}^{i}\right\}=\rho_{i} d t \quad \text { for } i=1,2 \text {, and } \mathbb{E}\left\{d B_{t}^{1} d B_{t}^{2}\right\}=\rho_{12} d t .
$$

The correlation coefficients are $\left|\rho_{1}\right|<1,\left|\rho_{2}\right|<1$ and $\left|\rho_{12}\right|<1$, satisfying $1+2 \rho_{1} \rho_{2} \rho_{12}-\rho_{1}^{2}-\rho_{2}^{2}-$ $\rho_{12}^{2}>0$. The parameters $\sigma, \varepsilon, \delta, \beta$ and $\eta$, the $\rho_{i}$ 's, and the long-term mean $\theta$, are all considered to be known. In most settings, $Y_{t}$ and $Z_{t}$ should not be considered observed because they must be estimated from market data, and hence they are filtered, making the investment problem one of partial information. In (2.1) $Y$ and $Z$ are both Ornstein-Uhlenbeck processes, hence the drift is Gaussian.

The expert opinions will give investors full information on $Z_{t}$ at time $t=0$. For instance, we can take $S_{t}$ to be the price of a managed fund whose performance is compared to a benchmark return of $\theta$, and excess returns on $S$ are given by $Y_{t}+Z_{t}$. We will consider an investment period of finite time $T$, and work in the regime $\frac{1}{\varepsilon} \gg T$ and $\frac{1}{\delta} \gg T$, which is interpreted below. Then returns from $Y$ are quickly averaged because $\varepsilon$ is small, and returns from $Z$ change very little for small $\delta$. Hence, the expert opinions are a prediction of $Z_{t}$ and will be more accurate for smaller $\delta$.

The information available to the investor at time $t \geq 0$ is contained in the filtration

$$
\mathcal{F}_{t} \triangleq \sigma\left\{S_{u}: 0 \leq u \leq t\right\}
$$


(i.e. the $\sigma$-algebra generated by the process $S$ up to time $t$ ). At time $t=0$, the expert opinions reveal the true value of $Z_{0}$ so that $\mathbb{E}\left\{Z_{0} \mid \mathcal{F}_{0}\right\}=Z_{0}$ and $\operatorname{var}\left\{Z_{0} \mid \mathcal{F}_{0}\right\}=0$. We could generalize the framework to have expert opinions arriving at multiple stochastic times, as done in [FGW12], but this paper will consider the basic case where expert opinions come at time $t=0$ only.

Given the role and effects of expert opinions, we are ready to provide an interpretation of the multiple time scales in $\left(Y_{t}, Z_{t}\right)$ :

i). $Y$ is a fast mean-reverting component in excess returns (e.g. intraday effects whose presence has been observed in the data [Pap13]). Experts do not give a value for $Y_{t}$ because the process changes far too fast for them to deliberate and announce their consensus.

ii). $Z$ is a slow mean-reverting component in excess returns. The slowness in the time scale allows the experts to carefully deliberate and deliver an accurate estimate of $Z_{0}$. The interpretation is that changes in $Z$ happen slowly, are not very large, and occur because of market events that the experts could not predict.

2.1. The Kalman Filter. For any square-integrable function $g: \mathbb{R} \times \mathbb{R} \rightarrow \mathbb{R}$, the posterior expectation is $\hat{g}_{t} \triangleq \mathbb{E}\left\{g\left(Y_{t}, Z_{t}\right) \mid \mathcal{F}_{t}\right\} \forall t \leq T$, and in particular we denote $\widehat{Y}_{t} \triangleq \mathbb{E}\left\{Y_{t} \mid \mathcal{F}_{t}\right\}$ and $\widehat{Z}_{t} \triangleq \mathbb{E}\left\{Z_{t} \mid \mathcal{F}_{t}\right\}$. Furthermore, we define the innovations process as

$$
\nu_{t} \triangleq \int_{0}^{t}\left(\frac{d S_{u}}{S_{u}}-\left(\theta+\widehat{Y}_{u}+\widehat{Z}_{u}\right) d u\right),
$$

which is an $\mathcal{F}_{t^{-}}$adapted Brownian motion if divided by the volatility $\sigma$. In (2.1), the drift of $S$ is Gaussian, which means that the Kalman-Bucy filter applies. In particular, the conditional distribution $\left(Y_{t}, Z_{t}\right) \mid \mathcal{F}_{t}$ is normal with covariance matrix

$$
\Sigma^{\varepsilon, \delta}(t) \triangleq \mathbb{E}\left(\begin{array}{c}
Y_{t}-\widehat{Y}_{t} \\
Z_{t}-\widehat{Z}_{t}
\end{array}\right)\left(\begin{array}{c}
Y_{t}-\widehat{Y}_{t} \\
Z_{t}-\widehat{Z}_{t}
\end{array}\right)^{\mathrm{T}}
$$

The definition of $\Sigma^{\varepsilon, \delta}$ has dropped the conditioning on $\mathcal{F}_{t}$ because $\left(\widehat{Y}_{t}, \widehat{Z}_{t}\right)$ is a Gaussian projection onto the observations $\left(S_{u}\right)_{u \leq t}$, and hence the residual difference $\left(Y_{t}-\widehat{Y}_{t}, Z_{t}-\widehat{Z}_{t}\right)$ is independent of the observations.

It follows that $\left(\widehat{Y}_{t}, \widehat{Z}_{t}\right)$ and $\Sigma^{\varepsilon, \delta}(t)$ are solutions of the following equations (see [BC09, Bre06] or [Kal80, Chapter10]):

$$
\begin{aligned}
d\left(\begin{array}{l}
\widehat{Y}_{t} \\
\widehat{Z}_{t}
\end{array}\right) & =-\left(\begin{array}{cc}
\frac{1}{\varepsilon} & 0 \\
0 & \delta
\end{array}\right)\left(\begin{array}{l}
\widehat{Y}_{t} \\
\widehat{Z}_{t}
\end{array}\right) d t+\frac{1}{\sigma^{2}} M^{\varepsilon, \delta}(t) d \nu_{t} \\
\frac{d}{d t} \Sigma^{\varepsilon, \delta}(t) & =-2\left(\begin{array}{cc}
\frac{1}{\varepsilon} & 0 \\
0 & \delta
\end{array}\right) \Sigma^{\varepsilon, \delta}(t)+\left(\begin{array}{cc}
\frac{\beta^{2}}{\varepsilon} & \rho_{12} \beta \eta \sqrt{\frac{\delta}{\varepsilon}} \\
\rho_{12} \beta \eta \sqrt{\frac{\delta}{\varepsilon}} & \eta^{2} \delta
\end{array}\right)-\frac{1}{\sigma^{2}} M^{\varepsilon, \delta}(t) M^{\varepsilon, \delta}(t)^{\mathrm{T}},
\end{aligned}
$$

where $M^{\varepsilon, \delta}(t)=\left(\Sigma^{\varepsilon, \delta}(t)\left(\begin{array}{l}1 \\ 1\end{array}\right)+\sigma\left(\begin{array}{c}\frac{\rho_{1} \beta}{\sqrt{\varepsilon}} \\ \rho_{2} \eta \sqrt{\delta}\end{array}\right)\right)$. Equations (2.4) and (2.5) are the Kalman-Bucy filter. We have that $\left(Y_{0}, Z_{0}\right) \mid \mathcal{F}_{0}$ is initially Gaussian distributed with moments $\widehat{Y}_{0}=\mathbb{E}\left\{Y_{0} \mid \mathcal{F}_{0}\right\}$, $\widehat{Z}_{0}=\mathbb{E}\left\{Z_{0} \mid \mathcal{F}_{0}\right\}$ and $\Sigma^{\varepsilon, \delta}(0)=\mathbb{E}\left(\begin{array}{c}Y_{0}-\widehat{Y}_{0} \\ Z_{0}-\widehat{Z}_{0}\end{array}\right)\left(\begin{array}{c}Y_{0}-\widehat{Y}_{0} \\ Z_{0}-\widehat{Z}_{0}\end{array}\right)^{\mathrm{T}}$. With the inclusion of expert opinions at time $t=0$, we have $\widehat{Z}_{0}=Z_{0}$ and $\Sigma^{\varepsilon, \delta}(0)=\left(\begin{array}{cc}\mathbb{E}\left(Y_{0}-\widehat{Y}_{0}\right)^{2} & 0 \\ 0 & 0\end{array}\right)$. 
2.2. Small- $\varepsilon$ Filter Asymptotics. In this section, we compute the limit of the posterior distribution on $\left(Y_{t}, Z_{t}\right)$ as $\varepsilon \rightarrow 0$. We derive an expansion of covariance matrix $\Sigma^{\varepsilon, \delta}(t)$ in powers of $\sqrt{\varepsilon}$, which will be used in Sections 4 and 5 to expand the investor's optimal value function.

Proposition 2.1. The covariance matrix $\Sigma^{\varepsilon, \delta}(t)$ that solves $(2.5)$ has a limit

$$
\Sigma^{\varepsilon, \delta}(t) \rightarrow \Sigma^{(0), \delta}(t) \triangleq\left(\begin{array}{cc}
\frac{\beta^{2}\left(1-\rho_{1}^{2}\right)}{2} & 0 \\
0 & \Sigma_{22}^{(0), \delta}(t)
\end{array}\right)
$$

as $\varepsilon \rightarrow 0$, where $\Sigma_{22}^{(0), \delta}(t)$ satisfies the Riccati equation

$$
\frac{d}{d t} \Sigma_{22}^{(0), \delta}(t)=-2 \delta\left(\Sigma_{22}^{(0), \delta}(t)-\frac{\eta^{2}\left(1-\rho_{2}^{2}\right)}{2}\right)-\frac{2 \eta \rho_{2} \sqrt{\delta}}{\sigma} \Sigma_{22}^{(0), \delta}(t)-\frac{1}{\sigma^{2}}\left(\Sigma_{22}^{(0), \delta}(t)\right)^{2},
$$

with initial condition $\Sigma_{22}^{(0), \delta}(0)=\mathbb{E}\left(Z_{0}-\widehat{Z}_{0}\right)^{2}$, and with $\Sigma_{22}^{(0)}=0$ when there are expert opinions.

The proof is given in Appendix A. The covariance matrix $\Sigma^{\varepsilon, \delta}(t)$ can also be expanded in powers of $\sqrt{\varepsilon}$ :

Proposition 2.2. Given the limit $\Sigma^{(0), \delta}(t)$ from Proposition 2.1, the solution $\Sigma^{\varepsilon, \delta}(t)$ to equation (2.5) can be expanded as

$$
\Sigma^{\varepsilon, \delta}(t)=\Sigma^{(0), \delta}(t)+\sqrt{\varepsilon} \Sigma^{(1), \delta}(t)+\ldots
$$

for $t>0$, where

$$
\Sigma^{(1), \delta}(t) \triangleq \frac{\rho_{1} \beta}{\sigma}\left(\begin{array}{cc}
\frac{\beta^{2}\left(1-\rho_{1}^{2}\right)}{2} & \Sigma_{22}^{(0), \delta}(t)+\rho_{2} \eta \sqrt{\delta} \\
\Sigma_{22}^{(0), \delta}(t)+\rho_{2} \eta \sqrt{\delta} & 0
\end{array}\right) .
$$

The proof is given in Appendix A.

2.3. Small- $\delta$ Asymptotics for $\Sigma_{22}^{(0), \delta}$ with Expert Opinions. The expert opinions give the investor full information on $Z_{0}$ at time $t=0$, that is

$$
\widehat{Z}_{0}=Z_{0} \quad \text { with } \quad \Sigma_{22}^{\varepsilon, \delta}(0)=0 .
$$

Hence, there is an explicit solution to equation (2.6), namely

$$
\Sigma_{22}^{(0), \delta}(t)=\left(\frac{1-e^{-d t}}{1-\frac{\alpha_{+}}{\alpha_{-}} e^{-d t}}\right) \alpha_{+}
$$

where $\alpha_{ \pm}$are the roots to the right-hand side in equation (2.6)

$$
\alpha_{ \pm}=-\left(\delta \sigma^{2}+\eta \rho_{2} \sqrt{\delta} \sigma\right) \pm \sqrt{\left(\delta \sigma^{2}+\eta \rho_{2} \sqrt{\delta} \sigma\right)^{2}+\delta \eta^{2} \sigma^{2}\left(1-\rho_{2}^{2}\right)},
$$

and $d=\left(\alpha_{+}-\alpha_{-}\right) / \sigma^{2}$.

Then for $\delta$ small we expand in powers of $\sqrt{\delta}$,

$$
\Sigma_{22}^{(0), \delta}(t)=\Sigma_{22}^{(0)}(t)+\sqrt{\delta} \Sigma_{22}^{(0,1)}(t)+\delta \Sigma_{22}^{(0,2)}+\ldots,
$$

where it is important to notice expert opinions at time $t=0$ cause the first two terms to be zero, namely, $\Sigma_{22}^{(0)}(t)=0=\Sigma_{22}^{(0,1)}(t)$ for all $t \geq 0$. Hence, $\Sigma_{22}^{(0), \delta}(t)=o(\sqrt{\delta})$. 
3. The Portfolio Problem with Partial Information. In the Merton problem, an investor allocates her capital dynamically over time between a risky stock and a riskless bank account to maximize expected utility of wealth at a fixed time horizon $T<\infty$. The investor has a self-financing wealth process $X_{t}$ satisfying

$$
d X_{t}=\pi_{t} \frac{d S_{t}}{S_{t}}+r\left(X_{t}-\pi_{t}\right) d t
$$

where $r \geq 0$ is the rate of return on wealth held in the risk-free bank account, and the process $\left(\pi_{t}\right)$ is the investor's control process adapted to $\mathcal{F}_{t}$ (defined in $(2.2)$ ), and satisfying the admissibility condition $\mathbb{E}\left\{\int_{0}^{T} \pi_{t}^{2} d t \mid \mathcal{F}_{0}\right\}<\infty$. Without loss of generality we take $r=0$ for the rest of the paper.

The investor's goal is to maximize terminal utility at time $T$,

$$
\sup _{\pi} \mathbb{E}\left\{U\left(X_{T}\right) \mid \mathcal{F}_{t} \vee\left\{X_{t}=x\right\}\right\} \quad \text { for } 0 \leq t \leq T<\infty
$$

where $U(x)$ is a smooth, increasing and strictly concave utility function on $\mathbb{R}^{+}$satisfying the Inada conditions $\lim _{x \rightarrow \infty} U^{\prime}(x)=0$ and $\lim _{x \searrow_{0}} U^{\prime}(x)=\infty$ (see, for instance, [KS98, KS99]). The difficulty in solving $(\mathcal{P})$ is the potentially non-Markovian nature of the problem. However, the Kalman filter $\left(\widehat{Y}_{t}, \widehat{Z}_{t}\right)$ encapsulates the information required from $\mathcal{F}_{t}$, and it is sufficient to write the value function for $(\mathcal{P})$ as the optimum of a Markov control problem:

$$
V^{\varepsilon, \delta}(t, x, y, z)=\sup _{\pi} \mathbb{E}\left\{U\left(X_{T}\right) \mid X_{t}=x, \widehat{Y}_{t}=y, \widehat{Z}_{t}=z\right\} .
$$

We will consider investment problem $\left(\mathcal{P}^{\prime}\right)$ for general utility functions. The reason for considering general utility functions is to allow for more realistic dependence of risk aversion on the level of investor wealth. For example, non-constant dependence on wealth is illustrated in [FSZ13] for the mixture of power utility functions.

From the definition of $\nu_{t}$ given in equation (2.3), we have a complete-markets description of the asset price

$$
\frac{d S_{t}}{S_{t}}=\left(\theta+\widehat{Y}_{t}+\widehat{Z}_{t}\right) d t+d \nu_{t} \quad \text { (observed price) }
$$

which allows us to rewrite the wealth process for $X_{t}$ and the filtering equations in (2.4) as

$$
\begin{aligned}
d X_{t} & =\pi_{t}\left(\theta+\widehat{Y}_{t}+\widehat{Z}_{t}\right) d t+\pi_{t} d \nu_{t} \\
d \widehat{Y}_{t} & =-\frac{1}{\varepsilon} \widehat{Y}_{t} d t+\frac{a^{\varepsilon, \delta}(t)}{\sigma} d \nu_{t} \\
d \widehat{Z}_{t} & =-\delta \widehat{Z}_{t} d t+\frac{b^{\varepsilon, \delta}(t)}{\sigma} d \nu_{t},
\end{aligned}
$$

where

$$
\begin{aligned}
& a^{\varepsilon, \delta}(t) \triangleq \frac{1}{\sigma}\left(\Sigma_{11}^{\varepsilon, \delta}(t)+\Sigma_{12}^{\varepsilon, \delta}(t)+\frac{\sigma \beta \rho_{1}}{\sqrt{\varepsilon}}\right) \\
& b^{\varepsilon, \delta}(t) \triangleq \frac{1}{\sigma}\left(\Sigma_{21}^{\varepsilon, \delta}(t)+\Sigma_{22}^{\varepsilon, \delta}(t)+\sqrt{\delta} \sigma \eta \rho_{2}\right) .
\end{aligned}
$$


The representation of $S_{t}$ in (3.1) is an SDE with $\mathcal{F}_{t}$-measurable coefficients and exactly one source of randomness (namely $\nu_{t}$ ), which means that the market is complete. Market completeness is important because in Section 3.2 it will lead to a linear PDE for the value function's convex dual. Of more immediate consequence from (3.1) is how these SDEs can be used to formulate a PDE to describe the value function in $\left(\mathcal{P}^{\prime}\right)$.

3.1. The HJB Equation. From the SDEs in (3.1), the optimal value function for $\left(\mathcal{P}^{\prime}\right)$ $V^{\varepsilon, \delta}(t, x, y, z)$ is associated with an HJB equation,

$$
\begin{aligned}
V_{t}^{\varepsilon, \delta}- & \frac{y}{\varepsilon} V_{y}^{\varepsilon, \delta}-\delta z V_{z}^{\varepsilon, \delta}+\frac{1}{2}\left(a^{\varepsilon, \delta}(t)\right)^{2} V_{y y}^{\varepsilon, \delta}+\frac{1}{2}\left(b^{\varepsilon, \delta}(t)\right)^{2} V_{z z}^{\varepsilon, \delta}+a^{\varepsilon, \delta}(t) b^{\varepsilon, \delta}(t) V_{y z}^{\varepsilon, \delta} \\
& +\max _{\pi}\left\{\frac{1}{2} \sigma^{2} \pi^{2} V_{x x}^{\varepsilon, \delta}+\pi\left((\theta+y+z) V_{x}^{\varepsilon, \delta}+\sigma\left(a^{\varepsilon, \delta}(t) V_{x y}^{\varepsilon, \delta}+b^{\varepsilon, \delta}(t) V_{x z}^{\varepsilon, \delta}\right)\right)\right\}=0,
\end{aligned}
$$

for all $t<T$, and with terminal condition $V^{\varepsilon, \delta}(T, x, y)=U(x)$ (see for instance [Pha09, Chapter3]). In general, regularity results for this type of fully-nonlinear HJB PDE problem are not available. However, we will show that small $\varepsilon$ is sufficient for smoothness of solutions. In addition, for some specific terminal conditions $U(x)$ such as power utility, explicit solutions are available (see [Bre06, KO96]).

From (3.4) we see that the optimal strategy is given in feedback form by

$$
\pi^{*}=-\frac{(\theta+y+z) V_{x}^{\varepsilon, \delta}+\sigma\left(a^{\varepsilon, \delta}(t) V_{x y}^{\varepsilon, \delta}+b^{\varepsilon, \delta}(t) V_{x z}^{\varepsilon, \delta}\right)}{\sigma^{2} V_{x x}^{\varepsilon, \delta}} .
$$

From this expression for optimal $\pi^{*}$ we see a decomposition of $\pi^{*}$ into a myopic strategy plus a correction for stochastic drift. Specifically, we have

$$
\text { myopic strategy }=-\frac{(\theta+y+z) V_{x}^{\varepsilon, \delta}}{\sigma^{2} V_{x x}^{\varepsilon, \delta}},
$$

and it is well known that the myopic strategy is optimal for logarithmic utility $U(x)=\log (x)$, but is sub-optimal for other utilities (see [BR05, SH04]). Indeed, plugging $\pi^{*}$ into (3.4) we have the following nonlinear PDE:

$$
\begin{aligned}
V_{t}^{\varepsilon, \delta}+\frac{1}{2}\left(a^{\varepsilon, \delta}(t)\right)^{2} V_{y y}^{\varepsilon, \delta}+a^{\varepsilon, \delta}(t) b^{\varepsilon, \delta}(t) V_{y z}^{\varepsilon, \delta}+\frac{1}{2}\left(b^{\varepsilon, \delta}(t)\right)^{2} V_{z z}^{\varepsilon, \delta}-\left(\frac{y}{\varepsilon} V_{y}^{\varepsilon, \delta}+\delta z V_{z}^{\varepsilon, \delta}\right) & \\
-\frac{\left(\frac{1}{\sigma}(\theta+y+z) V_{x}^{\varepsilon, \delta}+a^{\varepsilon, \delta}(t) V_{x y}^{\varepsilon, \delta}+b^{\varepsilon, \delta}(t) V_{x z}^{\varepsilon, \delta}\right)^{2}}{2 V_{x x}^{\varepsilon, \delta}} & =0,
\end{aligned}
$$

with terminal condition $V^{\varepsilon, \delta}(T, x, y, z)=U(x)$. The myopic strategy in (3.6) is optimal for logarithmic utility, because there is an additive separation of variables and cross terms $V_{x y}^{\varepsilon, \delta}$ and $V_{x z}^{\varepsilon, \delta}$ in (3.5) are zero (see [BR05]). However there is a non-myopic component for utilities such as HARA and CARA. Equation (3.7) for exponential and power utilities is central to the results in [Bre06, Car09]), and also for the case when the hidden process is a Markov chain in [BR05]. In this paper, for general concave utility functions we will use perturbation theory to find the terms in the formal expansion of the solution to (3.7),

$$
V^{\varepsilon, \delta}=V^{(0)}+\sqrt{\varepsilon} V^{(1,0)}+\sqrt{\delta} V^{(0,1)}+\ldots,
$$

however we will obtain these leading terms using convex duality. 
3.2. A Linear PDE for the Convex Dual. The nonlinearity in equation (3.7) does not carry over to the PDE for the Legendre transform of $V^{\varepsilon, \delta}$. The Legendre transform in the $x$-variable is

$$
\hat{V}^{\varepsilon, \delta}(t, p, y, z) \triangleq \sup _{x \in \mathbb{R}^{+}}\left(V^{\varepsilon, \delta}(t, x, y, z)-x p\right)
$$

for all $p>0$, for all $t \in[0, T]$, and for all $z, y \in \mathbb{R}$. For strictly concave $V^{\varepsilon, \delta}$ (with $\left.V_{x}\right|_{x=\infty}=0$ and $\left.V_{x}\right|_{x=0}=\infty$ due to the Inada conditions as shown in [KS99]) there is a 1-1 and onto mapping $x^{*}: \mathbb{R}^{+} \rightarrow \mathbb{R}^{+}$, such that for each $p$ the maximizer is the unique critical point $x^{*}=x^{*}(p)$ such that

$$
\hat{V}^{\varepsilon, \delta}(t, p, y, z)=V^{\varepsilon, \delta}\left(t, x^{*}(p), y, z\right)-p x^{*}(p) .
$$

As described in [JS02b], the partial derivatives of $V^{\varepsilon, \delta}$ evaluated at $x^{*}(p)$ have the following relationships with those of $\hat{V}^{\varepsilon, \delta}$ :

$$
\begin{aligned}
& \left.V_{x}^{\varepsilon, \delta}\right|_{x=x^{*}}=p \\
& \left.V_{x x}^{\varepsilon, \delta}\right|_{x=x^{*}}=-\frac{1}{\hat{V}_{p p}^{\varepsilon, \delta}} \\
& \left.V_{y y}^{\varepsilon, \delta}\right|_{x=x^{*}}=\frac{\hat{V}_{p p}^{\varepsilon, \delta} \hat{V}_{y y}^{\varepsilon, \delta}-\left(\hat{V}_{p y}^{\varepsilon, \delta}\right)^{2}}{\hat{V}_{p p}^{\varepsilon, \delta}} \\
& \left.V_{t}^{\varepsilon, \delta}\right|_{x=x^{*}}=\hat{V}_{t}^{\varepsilon, \delta} \\
& \left.V_{x y}^{\varepsilon, \delta}\right|_{x=x^{*}}=-\frac{\hat{V}_{p y}^{\varepsilon, \delta}}{\hat{V}_{p p}^{\varepsilon, \delta}} \\
& \left.V_{z z}^{\varepsilon, \delta}\right|_{x=x^{*}}=\frac{\hat{V}_{p p}^{\varepsilon, \delta} \hat{V}_{z z}^{\varepsilon, \delta}-\left(\hat{V}_{p z}^{\varepsilon, \delta}\right)^{2}}{\hat{V}_{p p}^{\varepsilon, \delta}} \\
& \left.V_{y}^{\varepsilon, \delta}\right|_{x=x^{*}}=\hat{V}_{y}^{\varepsilon, \delta} \\
& \left.V_{x z}^{\varepsilon, \delta}\right|_{x=x^{*}}=-\frac{\hat{V}_{p z}^{\varepsilon, \delta}}{\hat{V}_{p p}^{\varepsilon, \delta}} \\
& \left.V_{z y}^{\varepsilon, \delta}\right|_{x=x^{*}}=\frac{\hat{V}_{p p}^{\varepsilon, \delta} \hat{V}_{z y}^{\varepsilon, \delta}-\hat{V}_{p y}^{\varepsilon, \delta} \hat{V}_{p z}^{\varepsilon, \delta}}{\hat{V}_{p p}^{\varepsilon, \delta}} \\
& \left.V_{z}^{\varepsilon, \delta}\right|_{x=x^{*}}=\hat{V}_{z}^{\varepsilon, \delta} .
\end{aligned}
$$

When these partial derivatives are applied to equation (3.7), the effect is a PDE for the dual problem that is linear because the market is complete after filtering:

$$
\begin{aligned}
\hat{V}_{t}^{\varepsilon, \delta}+\frac{1}{2}\left(a^{\varepsilon, \delta}(t)\right)^{2} \hat{V}_{y y}^{\varepsilon, \delta}+a^{\varepsilon, \delta}(t) b^{\varepsilon, \delta}(t) \hat{V}_{y z}^{\varepsilon, \delta}+\frac{1}{2}\left(b^{\varepsilon, \delta}(t)\right)^{2} \hat{V}_{z z}^{\varepsilon, \delta}-\left(\frac{y}{\varepsilon} \hat{V}_{y}^{\varepsilon, \delta}+\delta z \hat{V}_{z}^{\varepsilon, \delta}\right) & \\
+\left(\frac{p^{2}(\theta+y+z)^{2}}{2 \sigma^{2}} \hat{V}_{p p}^{\varepsilon, \delta}-\frac{p(\theta+y+z)}{\sigma}\left(a^{\varepsilon, \delta}(t) \hat{V}_{p y}^{\varepsilon, \delta}+b^{\varepsilon, \delta}(t) \hat{V}_{p z}^{\varepsilon, \delta}\right)\right) & =0 \\
\hat{V}^{\varepsilon, \delta}(T, p, y, z) & =\hat{U}(p) .
\end{aligned}
$$

Here, $\hat{U}$ is the Legendre transform of the utility function $U$.

Equation (3.10) is degenerate, but existence of classical solutions is shown in Appendix B.1. In particular, it is shown that there exists $\varepsilon_{0}>0$ such that the Hörmander condition is satisfied for all $\varepsilon \leq \varepsilon_{0}$ (i.e. we prove regularity of solutions to the dual PDE under sufficiently fast speed of mean reversion in $Y$; small $\varepsilon$ has a regularizing effect). Moreover, this existence proof can be re-worked to show existence of solutions in the case when there is only a fast time scale, a result which pertains to [FPS14].

3.2.1. Obtaining a Solution to (3.7). Appendix B.1 has shown as that there exists smooth solutions to (3.10) for $\varepsilon$ small. In this brief subsection we explain how these solutions can be uniquely identified with the Feynman-Kac formula, and can be mapped back to the $x$-domain via 
convexity arguments. The arguments in this subsection will assume that $\varepsilon$ is sufficiently small so that we can be certain that smooth solutions exist.

The Feynman-Kac representation of the solution to (3.10) is

$$
\hat{V}^{\varepsilon, \delta}(t, p, y, z)=\mathbb{E}\left\{\hat{U}\left(p_{T}\right) \mid p_{t}=p, \widehat{Y}_{t}=y, \widehat{Z}_{t}=z\right\}
$$

where $p_{t}=p_{0} \exp \left\{-\frac{1}{2} \int_{0}^{t} \frac{\left(\theta+\widehat{Y}_{u}+\widehat{Z}_{u}\right)^{2}}{\sigma^{2}} d u-\int_{0}^{t} \frac{\theta+\widehat{Y}_{u}+\widehat{Z}_{u}}{\sigma^{2}} d \nu_{u}\right\}$, with $\nu_{t}$ being the same innovation Brownian process driving the SDEs in (3.1).

The inverse Legendre transform of $\hat{V}^{\varepsilon, \delta}$ gives the value function:

$$
\hat{\hat{V}}^{\varepsilon, \delta}(t, x, y, z) \triangleq \inf _{p>0}\left(\hat{V}^{\varepsilon, \delta}(t, p, y, z)+x p\right)=V^{\varepsilon, \delta}(t, x, y, z), \quad \forall x \in \mathbb{R}^{+} .
$$

4. Small- $\varepsilon$ Asymptotics. We define the operators

$$
\mathcal{D}_{k} \triangleq p^{k} \frac{\partial^{k}}{\partial p^{k}}
$$

for which we have commutativity (i.e. for any non-negative integers $k$ and $k$ ' we have $\mathcal{D}_{k} \mathcal{D}_{k^{\prime}}=$ $\mathcal{D}_{k^{\prime}} \mathcal{D}_{k}$ as follows easily from a change of variable to $\left.\log p\right)$.

Next we expand for $a^{\varepsilon, \delta}$ and $b^{\varepsilon, \delta}$ given by (3.2) and (3.3), respectively, in powers of $\sqrt{\varepsilon}$ using the expansion from Proposition 2.2. Then we use these expansions to rewrite equation (3.10) as

$$
\frac{1}{\varepsilon} \mathcal{L}_{0} \hat{V}^{\varepsilon, \delta}+\frac{1}{\sqrt{\varepsilon}} \mathcal{L}_{1}^{\delta} \hat{V}^{\varepsilon, \delta}+\mathcal{L}_{2}^{\delta} \hat{V}^{\varepsilon, \delta}+\sqrt{\varepsilon} \mathcal{L}_{3}^{\delta} \hat{V}^{\varepsilon, \delta}=o(\sqrt{\varepsilon}),
$$

where $o(\sqrt{\varepsilon})$ is the collection of higher-order terms coming from $o(\sqrt{\varepsilon})$ terms in the expansions of $a^{\varepsilon, \delta}$ and $b^{\varepsilon, \delta}$, and where the differential operators are defined as

$$
\begin{aligned}
& \mathcal{L}_{0} \triangleq \frac{\beta^{2} \rho_{1}^{2}}{2} \frac{\partial^{2}}{\partial y^{2}}-y \frac{\partial}{\partial y} \\
& \mathcal{L}_{1}^{\delta} \triangleq \beta \rho_{1}\left(\frac{1}{\sigma} \Sigma_{11}^{(0), \delta} \frac{\partial^{2}}{\partial y^{2}}+b^{\delta}(t) \frac{\partial^{2}}{\partial y \partial z}-\frac{\theta+y+z}{\sigma} \frac{\partial}{\partial y} \mathcal{D}_{1}\right) \\
& \mathcal{L}_{2}^{\delta} \triangleq \frac{\partial}{\partial t}-\delta z \frac{\partial}{\partial z}+\frac{1}{2}\left(b^{\delta}(t)\right)^{2} \frac{\partial^{2}}{\partial z^{2}}+\left(\frac{\rho_{1} \beta}{\sigma}\left(\Sigma_{12}^{(1), \delta}(t)+\Sigma_{11}^{(1), \delta}\right)+\frac{1}{2 \sigma^{2}}\left(\Sigma_{11}^{(0), \delta}\right)^{2}\right) \frac{\partial^{2}}{\partial y^{2}} \\
& \quad+\frac{(\theta+y+z)^{2}}{2 \sigma^{2}} \mathcal{D}_{2}-\frac{(\theta+y+z)}{\sigma}\left(b^{\delta}(t) \frac{\partial}{\partial z}+\frac{1}{\sigma} \Sigma_{11}^{(0), \delta} \frac{\partial}{\partial y}\right) \mathcal{D}_{1} \\
& \mathcal{L}_{3}^{\delta \triangleq} \triangleq\left(\frac{\rho_{1} \beta}{\sigma}\left(\Sigma_{12}^{(2), \delta}(t)+\Sigma_{11}^{(2), \delta}\right)+\frac{1}{\sigma^{2}} \Sigma_{11}^{(0), \delta}\left(\Sigma_{12}^{(1), \delta}(t)+\Sigma_{11}^{(1), \delta}\right)\right) \frac{\partial^{2}}{\partial y^{2}} \\
& \quad+\frac{\left(\Sigma_{12}^{(1), \delta}(t)+\Sigma_{11}^{(1), \delta}\right) b^{\delta}(t)+\sigma \beta \rho_{1} b^{(2), \delta}(t)}{\sigma} \frac{\partial^{2}}{\partial y \partial z}-\frac{(\theta+y+z)\left(\Sigma_{12}^{(1), \delta}(t)+\Sigma_{11}^{(1), \delta}\right)}{\sigma^{2}} \frac{\partial}{\partial y} \mathcal{D}_{1},
\end{aligned}
$$

with $b^{\delta}(t) \triangleq \frac{1}{\sigma}\left(\Sigma_{22}^{(0), \delta}(t)+\sqrt{\delta} \sigma \eta \rho_{2}\right), \Sigma_{11}^{(0), \delta}=\frac{\beta^{2}\left(1-\rho_{1}^{2}\right)}{2}$ (as given by Proposition 2.1), $\Sigma_{12}^{(1), \delta}(t)$ and $\Sigma_{11}^{(1), \delta}$ given by Proposition 2.2, and where $b^{(2), \delta}=\frac{1}{\sigma}\left(\Sigma_{21}^{(2), \delta}(t)+\Sigma_{22}^{(2), \delta}(t)+\sqrt{\delta} \sigma \eta \rho_{2}\right)$ with $\Sigma_{12}^{(2), \delta}(t), \Sigma_{11}^{(2), \delta}$ and $\Sigma_{22}^{(2), \delta}$ being the order- $\varepsilon$ terms that would come next in (2.7). 
We construct a formal expansion of the solution to equation (3.10) as

$$
\hat{V}^{\varepsilon, \delta}=\hat{V}^{(0), \delta}+\sqrt{\varepsilon} \hat{V}^{(1), \delta}+\varepsilon \hat{V}^{(2), \delta}+\varepsilon^{3 / 2} \hat{V}^{(3), \delta}+\ldots
$$

for $\varepsilon$ small. A useful notation for finding the terms in equation (4.2) is the bracket for $\mathcal{L}_{0}$ 's invariant average:

$$
\langle f\rangle \triangleq \int f(y) \mu(y) d y,
$$

for any square-integrable function $f(y)$, where $\mu(y)$ is the normal density function with mean zero and variance $\frac{1}{2} \rho_{1}^{2} \beta^{2}$.

4.1. The Order-Zero Term. Following the procedure of [FPSS11], we place the formal expansion (4.2) into equation (4.1) and collect terms of order $\varepsilon^{-1}$ to find $\mathcal{L}_{0} \hat{V}^{(0), \delta}=0$, which indicates $\hat{V}^{(0), \delta}$ is in the null space of $\mathcal{L}_{0}$ and therefore is constant in $y$. Then collecting terms of order $\varepsilon^{-1 / 2}$ we obtain $\mathcal{L}_{0} \hat{V}^{(1), \delta}=0$, and hence $\hat{V}^{(1), \delta}$ is also constant in $y$. Then comparing terms of order $\varepsilon^{0}$ we arrive at the equation

$$
\mathcal{L}_{0} \hat{V}^{(2), \delta}+\mathcal{L}_{2}^{\delta} \hat{V}^{(0), \delta}=0
$$

From the Fredholm alternative, we find that the solvability condition for $\hat{V}^{(2), \delta}$ in $(4.3)$ is

$$
\left\langle\mathcal{L}_{2}^{\delta} \hat{V}^{(0), \delta}\right\rangle=\overline{\mathcal{L}}_{2}^{\delta} \hat{V}^{(0), \delta}=0
$$

where

$$
\overline{\mathcal{L}}_{2}^{\delta} \triangleq \frac{\partial}{\partial t}+\frac{1}{2}\left(b^{\delta}(t)\right)^{2} \frac{\partial^{2}}{\partial z^{2}}-\delta z \frac{\partial}{\partial z}-\frac{(\theta+z) b^{\delta}(t)}{\sigma} \frac{\partial}{\partial z} \mathcal{D}_{1}+\frac{\left(\left\langle y^{2}\right\rangle+(\theta+z)^{2}\right)}{2 \sigma^{2}} \mathcal{D}_{2}
$$

using $\left\langle y^{2}\right\rangle=\int y^{2} \mu(y) d y=\frac{1}{2} \rho_{1}^{2} \beta^{2}$ and $\langle y\rangle=0$ (and the $y$ derivatives are left out because $\hat{V}^{(0), \delta}$ does not depend on $y$ ). The solvability condition then leads to the order-zero term in expansion (4.2) satisfying a Cauchy problem:

$$
\overline{\mathcal{L}}_{2}^{\delta} \hat{V}^{(0), \delta}=0, \quad \hat{V}^{(0), \delta}(T, p, z)=\hat{U}(p) .
$$

Equation (4.6) has strongly elliptic operator iff $\left\langle y^{2}\right\rangle \neq 0$, which is the case when $\rho_{1} \neq 0$.

4.2. The Order- $\sqrt{\varepsilon}$ Correction Term. Collecting terms of order $\sqrt{\varepsilon}$ in equation (4.1) and using the fact that $V_{y}^{(0), \delta}=V_{y y}^{(0), \delta}=0$, we arrive at the equation

$$
\mathcal{L}_{0} \hat{V}^{(3), \delta}+\mathcal{L}_{1}^{\delta} \hat{V}^{(2), \delta}+\mathcal{L}_{2}^{\delta} \hat{V}^{(1), \delta}=-\mathcal{L}_{3}^{\delta} \hat{V}^{(0), \delta}=0
$$

for which the solvability condition for $\hat{V}^{(3), \delta}$ is

$$
\left\langle\mathcal{L}_{1}^{\delta} \hat{V}^{(2), \delta}+\mathcal{L}_{2}^{\delta} \hat{V}^{(1), \delta}\right\rangle=0
$$

Since $\hat{V}^{(1), \delta}$ does not depend on $y$ we have $\left\langle\mathcal{L}_{2}^{\delta} \hat{V}^{(1), \delta}\right\rangle=\overline{\mathcal{L}}_{2}^{\delta} \hat{V}^{(1), \delta}$, and the solvability condition leads to

$$
\overline{\mathcal{L}}_{2}^{\delta} \hat{V}^{(1), \delta}=-\left\langle\mathcal{L}_{1}^{\delta} \hat{V}^{(2), \delta}\right\rangle=\left\langle\mathcal{L}_{1}^{\delta} \mathcal{L}_{0}^{-1}\left(\mathcal{L}_{2}^{\delta}-\overline{\mathcal{L}}_{2}^{\delta}\right) \hat{V}^{(0), \delta}\right\rangle
$$


where we have used equations (4.3) and (4.4) to replace $\hat{V}^{(2), \delta}$ inside the brackets. In this case, we have

$$
\left(\mathcal{L}_{2}^{\delta}-\overline{\mathcal{L}}_{2}^{\delta}\right) \hat{V}^{(0), \delta}=\left(\frac{y^{2}-\left\langle y^{2}\right\rangle+2 y(\theta+z)}{2 \sigma^{2}} \mathcal{D}_{2}-\frac{y b^{\delta}(t)}{\sigma} \frac{\partial}{\partial z} \mathcal{D}_{1}\right) \hat{V}^{(0), \delta}
$$

and we solve the following Poisson equations to obtain $\mathcal{L}_{0}^{-1}\left(\mathcal{L}_{2}^{\delta}-\left\langle\mathcal{L}_{2}^{\delta}\right\rangle\right) \hat{V}^{(0), \delta}$ :

$$
\begin{aligned}
\mathcal{L}_{0} \phi^{(1)}(y, z) & =\frac{y^{2}-\left\langle y^{2}\right\rangle+2 y(\theta+z)}{2 \sigma^{2}} \\
\mathcal{L}_{0} \phi^{(2)}(t, y) & =-\frac{y b^{\delta}(t)}{\sigma}
\end{aligned}
$$

which gives us

$$
\mathcal{L}_{0}^{-1}\left(\mathcal{L}_{2}^{\delta}-\left\langle\mathcal{L}_{2}^{\delta}\right\rangle\right) \hat{V}^{(0), \delta}=\left(\phi^{(1)}(y, z) \mathcal{D}_{2}+\phi^{(2)}(t, y) \frac{\partial}{\partial z} \mathcal{D}_{1}\right) \hat{V}^{(0), \delta}
$$

Hence, we have

$$
\begin{aligned}
\overline{\mathcal{L}}_{2}^{\delta} \hat{V}^{(1), \delta}= & \left\langle\mathcal{L}_{1}^{\delta}\left(\phi^{(1)}(\cdot, z) \mathcal{D}_{2}+\phi^{(2)}(t, \cdot) \frac{\partial}{\partial z} \mathcal{D}_{1}\right)\right\rangle \hat{V}^{(0), \delta} \\
= & \frac{\beta \rho_{1}}{\sigma} \Sigma_{11}^{(0), \delta}\left(\left\langle\phi_{y y}^{(1)}(\cdot, z)\right\rangle \mathcal{D}_{2}+\left\langle\phi_{y y}^{(2)}(t, \cdot)\right\rangle \frac{\partial^{2}}{\partial z^{2}} \mathcal{D}_{1}\right) \hat{V}^{(0), \delta} \\
& +\beta \rho_{1} b^{\delta}(t)\left(\left\langle\phi_{y}^{(1)}(\cdot, z)\right\rangle \frac{\partial}{\partial z} \mathcal{D}_{2}+\left\langle\phi_{y z}^{(1)}(\cdot, z)\right\rangle \mathcal{D}_{2}+\left\langle\phi_{y}^{(2)}(t, \cdot)\right\rangle \frac{\partial^{2}}{\partial z^{2}} \mathcal{D}_{1}\right) \hat{V}^{(0), \delta} \\
& -\frac{\beta \rho_{1}}{\sigma}\left((\theta+z)\left\langle\phi_{y}^{(1)}(\cdot, z)\right\rangle+\left\langle y \phi_{y}^{(1)}(\cdot, z)\right\rangle\right) \mathcal{D}_{1} \mathcal{D}_{2} \hat{V}^{(0), \delta} \\
& -\frac{\beta \rho_{1}}{\sigma}\left((\theta+z)\left\langle\phi_{y}^{(2)}(t, \cdot)\right\rangle+\left\langle y \phi_{y}^{(2)}(t, \cdot)\right\rangle\right) \frac{\partial}{\partial z} \mathcal{D}_{1}^{2} \hat{V}^{(0), \delta}
\end{aligned}
$$

Now notice that the solutions to equation (4.8) are given by $\phi^{(2)}(t, y)=\frac{y}{\sigma} b^{\delta}(t)+C(t, p, z)$, for some $C$ that does not depend on $y$. Therefore,

$$
\left\langle\phi_{y}^{(2)}(t, \cdot)\right\rangle=\frac{1}{\sigma} b^{\delta}(t), \quad\left\langle\phi_{y y}^{(2)}(t, \cdot)\right\rangle=0, \quad\left\langle y \phi_{y}^{(2)}(t, \cdot)\right\rangle=0 .
$$

Next, notice that $\frac{\beta^{2} \rho_{1}^{2}}{2} \mu_{y}(y)+y \mu(y)=0$, so that equation (4.7) can be re-written as

$$
\frac{\beta^{2} \rho_{1}^{2}}{2}\left(\phi_{y}^{(1)}(y, z) \mu(y)\right)_{y}=\frac{y^{2}-\left\langle y^{2}\right\rangle+2 y(\theta+z)}{2 \sigma^{2}} \mu(y) .
$$


Hence, $\phi_{y}^{(1)}(y, z)=\frac{2}{\beta^{2} \rho_{1}^{2} \mu(y)} \int_{-\infty}^{y} \frac{u^{2}-\left\langle y^{2}\right\rangle+2 u(\theta+z)}{2 \sigma^{2}} \mu(u) d u$. Then, similar to the calculations in [FPS14] and also the calculations on page 130 of [FPSS11], for $\phi^{(1)}$ we find

$$
\begin{gathered}
\left\langle\phi_{y}^{(1)}(\cdot, z)\right\rangle=\frac{2}{\beta^{2} \rho_{1}^{2}} \int_{-\infty}^{\infty}\left(\int_{-\infty}^{y} \frac{u^{2}-\left\langle y^{2}\right\rangle+2 u(\theta+z)}{2 \sigma^{2}} \mu(u) d u\right) d y=-\frac{\theta+z}{\sigma^{2}} \\
\left\langle y \phi_{y}^{(1)}(\cdot, z)\right\rangle=\frac{2}{\beta^{2} \rho_{1}^{2}} \int_{-\infty}^{\infty} y\left(\int_{-\infty}^{y} \frac{u^{2}-\left\langle y^{2}\right\rangle+2 u(\theta+z)}{2 \sigma^{2}} \mu(u) d u\right) d y=-\frac{\beta^{2} \rho_{1}^{2}}{4 \sigma^{2}},
\end{gathered}
$$

which also implies that $\left\langle\phi_{y z}^{(1)}(\cdot, z)\right\rangle=\frac{1}{2 \sigma^{2}}$. We also deduce $\left\langle\phi_{y y}^{(1)}(\cdot, z)\right\rangle=\frac{2}{\beta^{2} \rho_{1}^{2}}\left\langle y \phi_{y}^{(1)}(\cdot, z)\right\rangle=-\frac{1}{2 \sigma^{2}}$. Inserting these calculations into equation (4.9) yields the following equation for order- $\sqrt{\varepsilon}$ correction $\hat{V}^{(1), \delta}$ :

$$
\begin{aligned}
\overline{\mathcal{L}}_{2}^{\delta} \hat{V}^{(1), \delta}= & -\frac{\beta \rho_{1}}{2 \sigma^{3}} \Sigma_{11}^{(0), \delta} \mathcal{D}_{2} \hat{V}^{(0), \delta} \\
& +\beta \rho_{1} b^{\delta}(t)\left(-\frac{\theta+z}{\sigma^{2}} \frac{\partial}{\partial z} \mathcal{D}_{2}+\frac{1}{\sigma^{2}} \mathcal{D}_{2}+\frac{b^{\delta}(t)}{\sigma} \frac{\partial^{2}}{\partial z^{2}} \mathcal{D}_{1}\right) \hat{V}^{(0), \delta} \\
& +\frac{\beta \rho_{1}}{\sigma}\left(\frac{(\theta+z)^{2}}{\sigma^{2}}+\frac{\beta^{2} \rho_{1}^{2}}{4 \sigma^{2}}\right) \mathcal{D}_{1} \mathcal{D}_{2} \hat{V}^{(0), \delta}-\frac{\beta \rho_{1}}{\sigma} \frac{(\theta+z) b^{\delta}(t)}{\sigma} \frac{\partial}{\partial z} \mathcal{D}_{1}^{2} \hat{V}^{(0), \delta} \\
\hat{V}^{(1), \delta}(T, x, z)= & 0
\end{aligned}
$$

Solving equation (4.10) is not a simple computation, but will simplify considerably in Section 5.3 when we consider small- $\delta$ behavior .

5. Small- $\delta$ Asymptotics Using Expert Opinions. Let's now consider expert opinions so that $\widehat{Z}_{0}=Z_{0}$ with initial covariance $\Sigma_{22}^{(0), \delta}(0)=0$, and for time $t>0$ having covariance $\Sigma_{22}^{(0), \delta}(t)$ with small- $\delta$ expansion given by equation (2.8). Sections 5.1 and 5.2 will expand $\hat{V}^{(0), \delta}$ from Section 4.1 as

$$
\hat{V}^{(0), \delta}=\hat{V}^{(0)}+\sqrt{\delta} \hat{V}^{(0,1)}+\delta \hat{V}^{(0,2)}+\ldots
$$

and will compute the order-zero and order- $\sqrt{\delta}$ terms, respectively. Then, Section 5.3 will compute the order- $\sqrt{\varepsilon}$ correction term that is the limit of $\hat{V}^{(1), \delta}$ that solves $(4.10)$.

We start with the following definition:

DEFINITION 5.1. The risk tolerance function based on the order-zero value function is defined as

$$
R^{(0)}(t, x, z) \triangleq-\frac{V_{x}^{(0)}(t, x, z)}{V_{x x}^{(0)}(t, x, z)} .
$$

In general, the value function $V^{(0)}(t, x, z)$ inherits the properties of the utility $U(x)$, specifically it is increasing and strictly concave in the wealth level $x$. The quantity $-\frac{U^{\prime \prime}}{U^{\prime}}$ is classically known as the Arrow-Pratt measure of absolute risk aversion (see for instance [HL88, Chapter1]). The risk tolerance given in Definition 5.1 is simply the reciprocal of this measure using the value function (or indirect utility) $V^{(0)}$. 
5.1. The Order-Zero Term. The order-zero term in equation (5.1) is the small- $\delta$ limit of the solution to equation (4.6). To obtain this limit, we start by considering the limiting behavior of the differential operator defined in equation (4.5), which is

$$
\overline{\mathcal{L}}_{2}^{(0)} \triangleq \frac{\partial}{\partial t}+\frac{1}{2} \bar{\lambda}^{2}(z) \mathcal{D}_{2}
$$

where $\bar{\lambda}(z) \triangleq \sqrt{\frac{\beta^{2} \rho_{1}^{2}}{2 \sigma^{2}}+\frac{(\theta+z)^{2}}{\sigma^{2}}}$. This limit is easily deduced by recognizing that $b^{\delta}(t)$ goes to zero as $\delta \rightarrow 0$, and so $\overline{\mathcal{L}}_{2}^{(0)}$ is simply the remaining terms. Indeed, if we plug the expansion of (5.1) into equation (4.6) for $\hat{V}^{(0), \delta}$, then collecting terms of order $\delta^{0}$ we find that the order-zero term in (5.1) solves the Cauchy problem

$$
\overline{\mathcal{L}}_{2}^{(0)} \hat{V}^{(0)}=0, \quad \hat{V}^{(0)}(T, p, z)=\hat{U}(p) .
$$

Furthermore, we can transform the PDE for the dual solution back to PDE in the primal domain. Let us define the inverse dual

$$
V^{(0)}(t, x, z) \triangleq \inf _{p \geq 0}\left(\hat{V}^{(0)}(t, p, z)+x p\right) .
$$

Applying the dual transforms of (3.9) we can convert the dual differential equation in (5.3) into its primal version,

$$
\mathcal{D}_{2} \hat{V}^{(0)}=p^{2} \hat{V}_{p p}^{(0)}=-\left.\frac{V_{x}^{(0)}}{V_{x x}^{(0)}} V_{x}^{(0)}\right|_{x=x *}=\left.R^{(0)} V_{x}^{(0)}\right|_{x=x *}=-\left.\left(R^{(0)}\right)^{2} V_{x x}^{(0)}\right|_{x=x *},
$$

where $x^{*}=x^{*}(p)$ is the unique critical point such that

$$
\hat{V}^{(0)}(t, p, z)=\sup _{x \geq 0}\left(V^{(0)}(t, x, z)-x p\right)=V^{(0)}\left(t, x^{*}, z\right)-x^{*} p .
$$

Hence the order-zero term in the $\varepsilon$ and $\delta$ expansion of the primal value function is the solution to

$$
\left(\frac{\partial}{\partial t}-\frac{1}{2} \bar{\lambda}^{2}(z)\left(R^{(0)}\right)^{2} \frac{\partial^{2}}{\partial x^{2}}\right) V^{(0)}=0, \quad V^{(0)}(T, x, z)=U(x) .
$$

5.2. The Order $-\sqrt{\delta}$ Correction Term. To obtain equation (5.3) we collected terms of order $\delta^{0}$. After inserting the expansion of (5.1) into equation (4.6) and applying the small- $\delta$ expansion of equation (2.8), we collect the terms of order $\sqrt{\delta}$ to obtain the following PDE problem for the order- $\sqrt{\delta}$ correction:

$$
\overline{\mathcal{L}}_{2}^{(0)} \hat{V}^{(0,1)}=\rho_{2} \eta \frac{\theta+z}{\sigma} \frac{\partial}{\partial z} \mathcal{D}_{1} \hat{V}^{(0)}, \quad \hat{V}^{(0,1)}(T, p, z)=0 .
$$

Due to the commutativity of the $\mathcal{D}_{k}$ operators, there is an explicit solution to (5.6):

Proposition 5.2. Equation (5.6) has the explicit solution

$$
\hat{V}^{(0,1)}(t, p, z)=-(T-t)^{2} \rho_{2} \eta \frac{(\theta+z)^{2}}{2 \sigma^{3}} \mathcal{D}_{2} \mathcal{D}_{1} \hat{V}^{(0)}(t, p, z)=-(T-t) \rho_{2} \eta \frac{\theta+z}{2 \sigma} \frac{\partial}{\partial z} \mathcal{D}_{1} \hat{V}^{(0)}(t, p, z) .
$$


Proof. This follows from calculations similar to those in the proof of [FSZ13, Proposition 3.2]).

Furthermore, we can use the duality relations of (3.9) to transform the explicit expression in (5.7) back into the order- $\sqrt{\delta}$ correction in the formal expansion proposed in equation (3.8),

Proposition 5.3. In the primal formulation, the order $-\sqrt{\delta}$ correction in the singular-regular expansion of $V^{\varepsilon, \delta}$ in equation (3.8) is given explicitly as

$$
V^{(0,1)}(t, x, z)=(T-t) \rho_{2} \eta \frac{\theta+z}{2 \sigma} R^{(0)}(t, x, z) \frac{\partial^{2}}{\partial z \partial x} V^{(0)}(t, x, z) .
$$

Proof. Applying the the duality relations of (3.9) to the convex, zero-order value function $V^{(0)}$, to see that

$$
\frac{\partial}{\partial z} \mathcal{D}_{1} \hat{V}^{(0)}=p \hat{V}_{p z}^{(0)}=\left.\frac{V_{x}^{(0)}}{V_{x x}^{(0)}} V_{x z}^{(0)}\right|_{x=x *}=-\left.R^{(0)} V_{x z}^{(0)}\right|_{x=x^{*}},
$$

where $x^{*}=x^{*}(p)$ is the unique critical point defined in (5.4). We use this to transform (5.7) back into the primal domain, and we obtain the result.

5.3. Limit of the Order- $\sqrt{\varepsilon}$ Correction Term. As $\delta$ tends toward zero, the solution to equation (4.10) can be expanded in powers of $\sqrt{\delta}$,

$$
\hat{V}^{(1), \delta}=\hat{V}^{(1,0)}+\sqrt{\delta} \hat{V}^{(1,1)}+\delta \hat{V}^{(1,2)}+\ldots .
$$

The order-zero term in this expansion is the order- $\sqrt{\varepsilon}$ expansion to $\hat{V}^{\varepsilon, \delta}$ for small $\varepsilon$ and $\delta$. Indeed, plugging the expansion of (5.9) into equation (4.10) and collecting terms of order $\delta^{0}$, we find that $\hat{V}^{(1,0)}$ satisfies the following PDE:

$$
\overline{\mathcal{L}}_{2}^{(0)} \hat{V}^{(1,0)}=-\left(\frac{\beta \rho_{1}}{2 \sigma^{3}} \Sigma_{11}^{(0), \delta} \mathcal{D}_{2}-\frac{\beta \rho_{1}}{\sigma}\left(\frac{(\theta+z)^{2}}{\sigma^{2}}+\frac{\beta^{2} \rho_{1}^{2}}{4 \sigma^{2}}\right) \mathcal{D}_{1} \mathcal{D}_{2}\right) \hat{V}^{(0)},\left.\quad \hat{V}^{(1,0)}\right|_{t=T}=0 .
$$

Then from the commutativity of the $\mathcal{D}_{k}$ operators it is easy to obtain an explicit solution:

Proposition 5.4. The explicit solution to (5.10) is given by

$$
\hat{V}^{(1,0)}(t, p, z)=(T-t)\left(\frac{\beta^{3} \rho_{1}\left(1-\rho_{1}^{2}\right)}{4 \sigma^{3}} \mathcal{D}_{2}-\frac{\beta \rho_{1}}{\sigma}\left(\frac{(\theta+z)^{2}}{\sigma^{2}}+\frac{\beta^{2} \rho_{1}^{2}}{4 \sigma^{2}}\right) \mathcal{D}_{1} \mathcal{D}_{2}\right) \hat{V}^{(0)}(t, p, z) .
$$

Proof. The operators $\mathcal{D}_{k}$ and $\mathcal{D}_{k^{\prime}}$ commute for different $k \neq k^{\prime}$, hence it's straightforward to check that (5.11) is the solution.

Applying the dual transforms in (3.9), along with an identity for $\hat{V}_{p p p}^{(0)}$, the dual solution in (5.11) is transformed back to obtaib the order- $\sqrt{\varepsilon}$ term of the formal expansion proposed in (3.8):

PROPOSITION 5.5. In the primal formulation, the order- $\sqrt{\varepsilon}$ correction in the singular-regular expansion of $V^{\varepsilon, \delta}$ in equation (3.8) is given explicitly as

$V^{(1,0)}(t, x, z)=(T-t)\left(\frac{\beta^{3} \rho_{1}\left(1-\rho_{1}^{2}\right)}{4 \sigma^{3}} R^{(0)} \frac{\partial}{\partial x}+\frac{\beta \rho_{1}}{\sigma}\left(\frac{(\theta+z)^{2}}{\sigma^{2}}+\frac{\beta^{2} \rho_{1}^{2}}{4 \sigma^{2}}\right)\left(R^{(0)} \frac{\partial}{\partial x}\right)^{2}\right) V^{(0)}(t, x, z)$. 
Proof. Applying the dual transforms in (3.9), along with the identity that $\hat{V}_{p p p}^{(0)}=\left.\frac{V_{x x}^{(0)}}{\left(V_{x x}^{(0)}\right)^{3}}\right|_{x=x^{*}}$ we find that

$$
\mathcal{D}_{1} \mathcal{D}_{2} \hat{V}^{(0)}=2 p^{2} \hat{V}_{p p}^{(0)}+p^{3} \hat{V}_{p p p}^{(0)}=\left(-2 \frac{\left(V_{x}^{(0)}\right)^{2}}{V_{x x}^{(0)}}+\left.\frac{V_{x x x}^{(0)}\left(V_{x}^{(0)}\right)^{3}}{\left(V_{x x}^{(0)}\right)^{3}}\right|_{x=x^{*}}=-\left.\left(R^{(0)} \frac{\partial}{\partial x}\right)^{2} V^{(0)}\right|_{x=x^{*}},\right.
$$

where $x^{*}=x^{*}(p)$ is the unique critical point in (5.4). This shows how the dual solution in (5.11) is transformed back into the primal domain to obtain the result.

REMARK 1. The expansions of $V^{\varepsilon, \delta}$ solving the nonlinear HJB equation for small $\varepsilon$ and small $\delta$ with expert opinions, as proposed in equation (3.8), is

$$
V^{\varepsilon, \delta}=V^{(0)}+\sqrt{\varepsilon} V^{(1,0)}+\sqrt{\delta} V^{(0,1)}+o(\sqrt{\varepsilon})+o(\sqrt{\delta})
$$

where $V^{(0)}, V^{(1,0)}$ and $V^{(0,1)}$ are given by (5.5), (5.8), and (5.12), respectively. Some remarks on the accuracy of this final expansion of solutions to the nonlinear HJB equation are given in Appendix B.2.

6. Numerical Studies. Our numerical studies aim for understanding of the small- $\delta$ partialinformation effects in the small- $\varepsilon$ limit. We consider the parameterization $\theta=0, \sigma=.15, \delta=.05$, $\beta=.2, \rho_{1}=-.3, \eta=.3$, and $\rho_{2}=-.7$. We don't specify a parameter $\varepsilon$, but simply assume that it is small. We consider the mixture of power utility function

$$
U(x)=\frac{c_{1}}{1-\gamma_{1}} x^{1-\gamma_{1}}+\frac{c_{2}}{1-\gamma_{2}} x^{1-\gamma_{2}}
$$

with $c_{1}=c_{2}=.5$ and $\gamma_{2}=.45, \gamma_{1}=.9$.

6.1. Partial Information Expansion Using Black's Equation. As shown in [FSZ13], it turns out that the solution $V^{(0)}$ to equation (5.5) can be obtained using the so-called Black's PDE for $R^{(0)}$, where $R^{(0)}$ is the risk-tolerance function defined in equation (5.2). The PDE for $R^{(0)}$ is

$$
\begin{aligned}
R_{t}^{(0)}+\frac{1}{2} \bar{\lambda}^{2}(z)\left(R^{(0)}\right)^{2} R_{x x}^{(0)} & =0 \quad \text { for } t<T, \\
R^{(0)}(T, x) & =-\frac{U_{x}(x)}{U_{x x}(x)},
\end{aligned}
$$

from which it can be determined that $V_{x}^{(0)}$ is given by the following formula:

$$
V_{x}^{(0)}(t, x, z)=V_{x}^{(0)}\left(t, x_{\max }, z\right) \exp \left(\int_{x}^{x_{\max }} \frac{1}{R^{(0)}(t, \xi, z)} d \xi\right),
$$

where $x_{\max } \gg 0$ is some high value of the wealth process (e.g. $x_{\max }$ is the upper boundary on a numerical domain). The recipe for $V^{(0)}$ is to solve for $R^{(0)}$, integrate to get $V_{x}^{(0)}$, and then integrate over $x$ to get $V^{(0)}$,

$$
V^{(0)}(t, x, z)=V^{(0)}\left(t, x_{\max }, z\right)-\int_{x}^{x_{\max }} V_{x}^{(0)}(t, \xi, z) d \xi
$$


For mixed power utility, the large- $x$ asymptotics are given in [FSZ13]. When solving numerically for the mixed power utility, we use the solution $R^{(0)}$ 's asymptotic behavior of $\lim _{x \backslash 0} R^{(0)}(t, x, z)=0$ and $\lim _{x \nearrow_{\infty}} R_{x}^{(0)}(t, x, z)=\frac{1}{\gamma_{2}}$ as numerical boundary conditions. Given $V^{(0)}$, we then compute $V^{(0,1)}(t,, x, z)$ based on formula in equation (5.8). Figure 6.1 (left plot) shows the value-function approximation $V^{(0)}+\sqrt{\delta} V^{(0,1)}$.
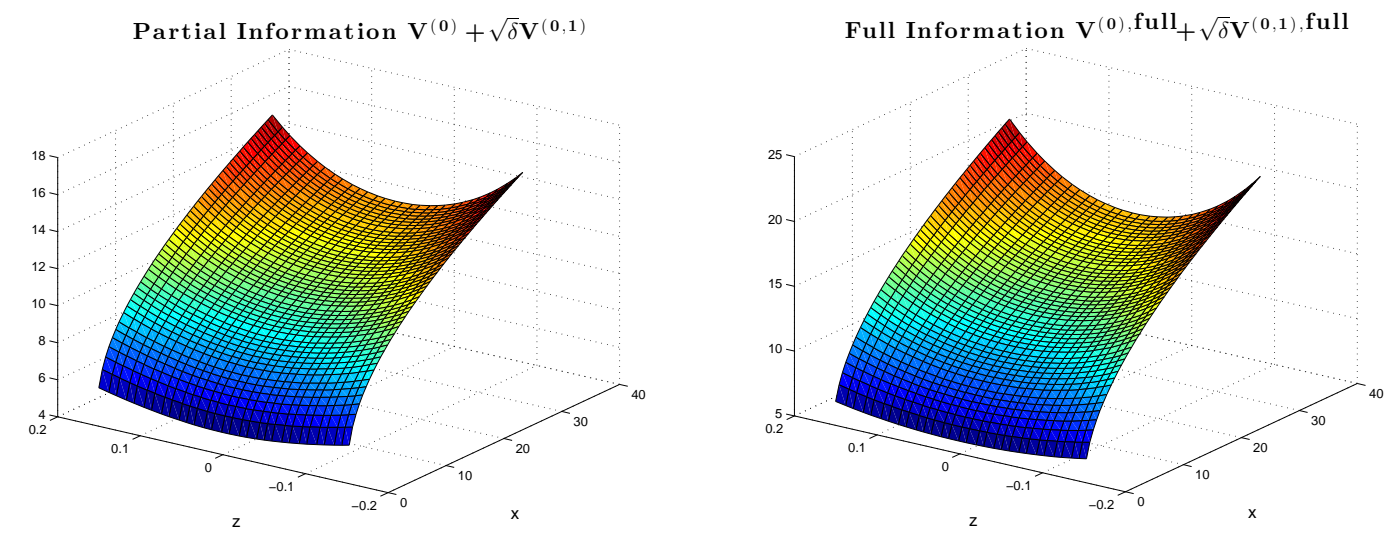

FIG. 6.1. The value-function expansion $V^{(0)}+\sqrt{\delta} V^{(0,1)}$ (left) and $V^{(0), \delta, f u l l}$ (right) with the parameters given at the beginning of Section 6. The numerical scheme uses time step $\Delta t=.02, T=1, \Delta z=.003$, $\Delta x=0.0455$, and evaluated at time $t=0$.

6.2. The Full-Information Case. This section will provide a brief review of the method for computing the full-information value function, and will highlight some qualitative differences/similarities to partial information. The full-information problem is

$$
V^{\varepsilon, \delta, \text { full }}(t, x, y, z)=\sup _{\pi} \mathbb{E}\left\{U\left(X_{T}\right) \mid X_{t}=x, Y_{t}=y, Z_{t}=z\right\},
$$

with optimal value function $V^{\varepsilon, \delta \text {,full }}$ that solves the following full-information HJB equation

$$
\begin{aligned}
V_{t}^{\varepsilon, \delta, \text { full }}+\frac{1}{\varepsilon} \mathcal{L}_{0} V^{\varepsilon, \delta, \text { full }}+\delta \mathcal{M} V^{\varepsilon, \delta, \text { full }}+\rho_{12} \beta \eta \sqrt{\frac{\delta}{\varepsilon}} V_{z y}^{\varepsilon, \delta \text { full }} \\
-\frac{\left(\frac{\theta+y+z}{\sigma} V_{x}^{\varepsilon, \delta \text { full }}+\frac{\beta \rho_{1}}{\sqrt{\varepsilon}} V_{x y}^{\varepsilon, \delta \text { full }}+\eta \rho_{2} \sqrt{\delta} V_{x z}^{\varepsilon, \delta \text { full }}\right)^{2}}{2 V_{x x}^{\varepsilon, \delta \text { full }}}=0,
\end{aligned}
$$

where $\mathcal{L}_{0} \triangleq \frac{\beta^{2}}{2} \frac{\partial^{2}}{\partial y^{2}}-y \frac{\partial}{\partial y}$ and $\mathcal{M} \triangleq \frac{\eta^{2}}{2} \frac{\partial^{2}}{\partial z^{2}}-z \frac{\partial}{\partial z}$, with terminal condition $V^{\varepsilon, \delta \text { full }}(T, x, y)=U(x)$. Due to strong ellipticity of the infinitesimal generator to the full-information SDEs, we expect there to exist strong solutions to (6.3), particularly for small $\varepsilon$ and $\delta$ when the solution will be very close to the standard Merton value function. Furthermore, HARA and CARA utilities allow (6.3) to be solved with an ansatz that yields explicit solutions, such as those given in [Bre06].

We write an expansion of the solution to (6.3),

$$
V^{\varepsilon, \delta, \text { full }}=V^{(0), \text { full }}+\sqrt{\delta} V^{(0,1), \text { full }}+\sqrt{\varepsilon} V^{(1,0), \text { full }}+o(\sqrt{\varepsilon})+o(\sqrt{\delta}),
$$


where the terms $V^{(0), \text { full }}$ and $V^{(0,1) \text {,full }}$ are computed using the asymptotic formulas from [FSZ13]. In particular, neither $V^{(0) \text {,full }}$ or $V^{(0,1) \text {,full }}$ depend on $y$ :

$$
V_{t}^{(0), \text { full }}+\frac{1}{2} \bar{\lambda}_{\mathrm{f}}^{2}(z) \frac{\left(V_{x}^{(0), \text { full }}\right)^{2}}{V_{x x}^{(0), \text { full }}}=0 \quad \text { for } t<T,
$$

where $\bar{\lambda}_{\mathrm{f}}(z) \triangleq \sqrt{\frac{\beta^{2}}{2 \sigma^{2}}+\frac{(\theta+z)^{2}}{\sigma^{2}}}$, with terminal condition $V^{(0), \text { full }}(T, x)=U(x)$, and

$$
V^{(0,1), \text { full }}=-(T-t) \frac{\rho_{2} \eta(\theta+z)}{2 \sigma} \frac{V_{x}^{(0), \text { full }}}{V_{x x}^{(0), \text { full }}} V_{x z}^{(0), \text { full }} \quad \text { for } t \leq T .
$$

Similar to the partial-information case in Section 6.1, there is a full-information risk-tolerance

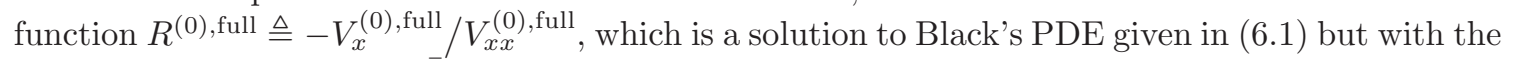
full-information coefficient $\bar{\lambda}_{\mathrm{f}}(z)$. Figure 6.1 (right plot) shows the full-information value-function approximation $V^{(0), \delta \text {,full }} \approx V^{(0) \text {,full }}+\sqrt{\delta} V^{(0,1), \text { full }}$, computed based on the full-information version of Black's equation.

6.3. Comparisons with Full Information. In [Bre06], [FPS14] and [Pap13], comparison between partial-information and full-information value functions has shown there to be an information premium. In particular, investors experience a lowering of their value function when the market is not fully observable. In this section we compare and contrast the order-zero and order- $\sqrt{\delta}$ terms, for both partial and full information, and explain how these terms capture the additional risk associated with stochastic excess returns and partial information.

The information premium in the order-zero term is highlighted in [FPS14], where the partial information Sharpe ratio was significantly lower than that of full information, with $\rho_{1}$ being the parameter with the biggest impact. Namely, the partial-information Sharpe ratio $\bar{\lambda}(z)$ defined in Section 5.1 and for full-information $\bar{\lambda}_{f}(z)$ defined in Section 6.2 have the relationship

$$
\bar{\lambda}(z)<\bar{\lambda}_{f}(z) \quad \text { iff } \quad\left|\rho_{1}\right|<1 .
$$

We have taken $\rho_{1}=-.3$ in the numerical examples of this paper, and we can visually tell that there is decreased Sharpe ratio in partial information plots of Figure 6.2. The full-information order-zero

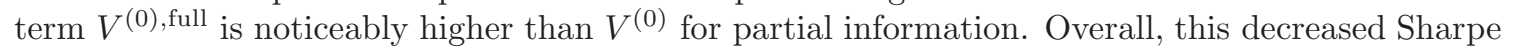
ratio can be thought of as an information premium due to partial information because having $\rho_{1}= \pm 1$ is equivalent having fully-observable $Y_{t}$, in which case we have $\bar{\lambda}(z)=\bar{\lambda}_{f}(z)$.

The effects of the order- $\sqrt{\delta}$ corrections are more qualitative. In Figure 6.2 we can see that the order- $\sqrt{\delta}$ correction has a lowering effect on the value function, which is due in large part to the parameterization of $\rho_{2}<0$. For the full-information value function, it should be clear that the order- $\sqrt{\delta}$ term is correcting for the additional risk due to stochasticity in excess returns, yet it is interesting to see that the corrected full-information value function is still higher than the order-zero term for partial-information, that is $V^{(0), \text { full }}+\sqrt{\delta} V^{(0,1), \text { full }}>V^{(0)}$.

For the partial information value function, it is difficult to differentiate an information premium from a premium on risk from stochasticity in excess returns. From the top-row plots in Figure 6.2 we see that $V^{(0)}+\sqrt{\delta} V^{(0,1)}<V^{(0)}$, but this correction is due to stochasticity in $\widehat{Z}_{t}$, whereas the full-information correction was a correction due to stochasticity in $Z_{t}$. Indeed, we can see a further comparison of the full and partial correction in Figure 6.3, where the expansions are compared for 


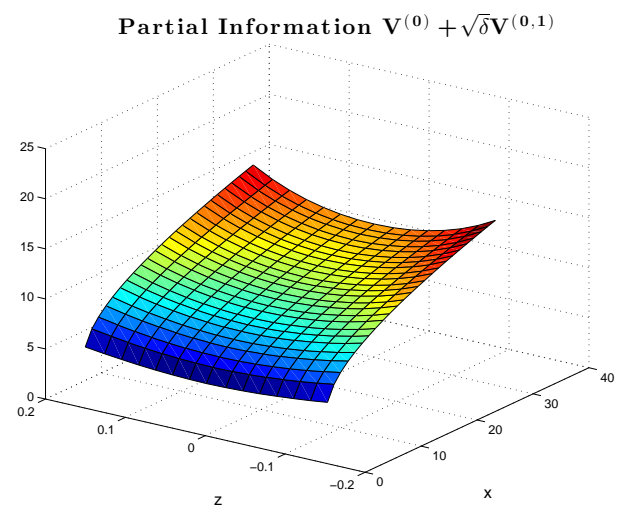

Full Information $\mathbf{V}^{(0), \text { full }}+\sqrt{\delta} \mathbf{V}^{(0,1), \text { full }}$

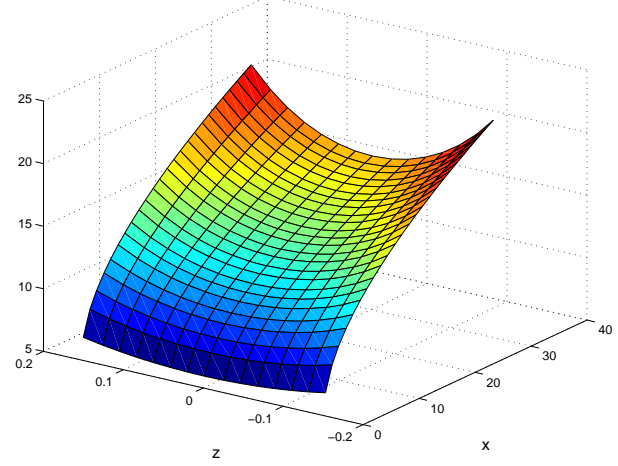

Partial Information $\mathbf{V}^{(0)}$

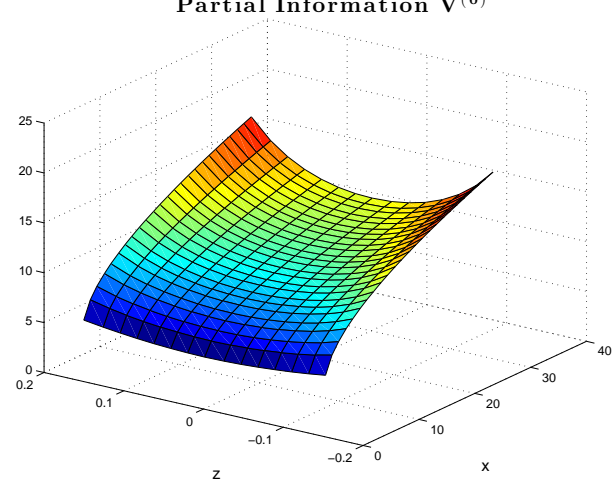

Full Information $\mathbf{V}^{(0)}$, full

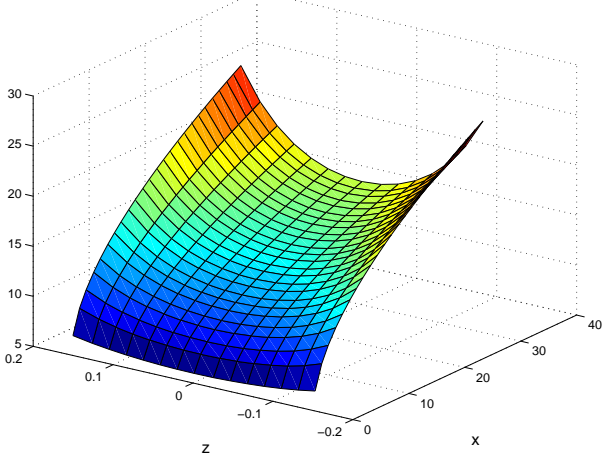

FIg. 6.2. Top Left: The partial-information approximation $V^{(0)}+\sqrt{\delta} V^{(0,1)}$. Top Right: The orderzero term $V^{(0)}$, which corresponds to the order-zero term under partial information with expert opinions.

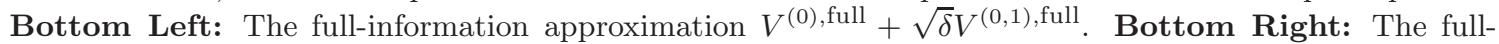

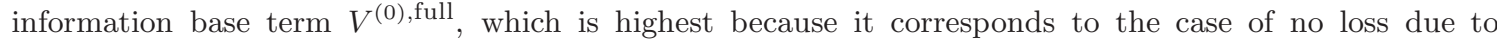
stochastic drift.

various levels of $z$. An important thing to notice from the plotted lines is that the correction to the partial-information value function is less than the correction for full-information, which can be attributed to the fact that the process $\widehat{Z}_{t}$ has much less volatility than the observed $Z_{t}$.

6.4. Noise in Expert Opinions. In this section we consider an HJB equation that corresponds to the setting where the expert opinion is a noisy unbiased estimate of $Z_{0}$ with standard deviation that is of order- $\sqrt{\delta}$, that is $\sqrt{\Sigma_{22}^{(0), \delta}(0)} \sim \sqrt{\delta}$ and $\widehat{Z}_{0} \sim N\left(Z_{0}, \Sigma_{22}^{(0), \delta}(0)\right)$. We will compute a numerical solution to the PDE for $\hat{V}^{(0), \delta}$ with a scheme taking full consideration of $\delta>0$ effects, and then compare with the small- $\delta$ expansions of Section 5 where the expert opinion was noiseless. From looking at numerical solutions we will see that expert opinions don't make a difference unless they have noise that is smaller than order $\sqrt{\delta}$.

To make the computations simpler we consider a case where the PDE coefficients are constant in time. Suppose that $\widehat{Z}^{(0)}$ will be in its invariant state. That is, suppose that $\Sigma_{22}^{(0), \delta}(0)=\bar{\Sigma}^{\delta}$ where 

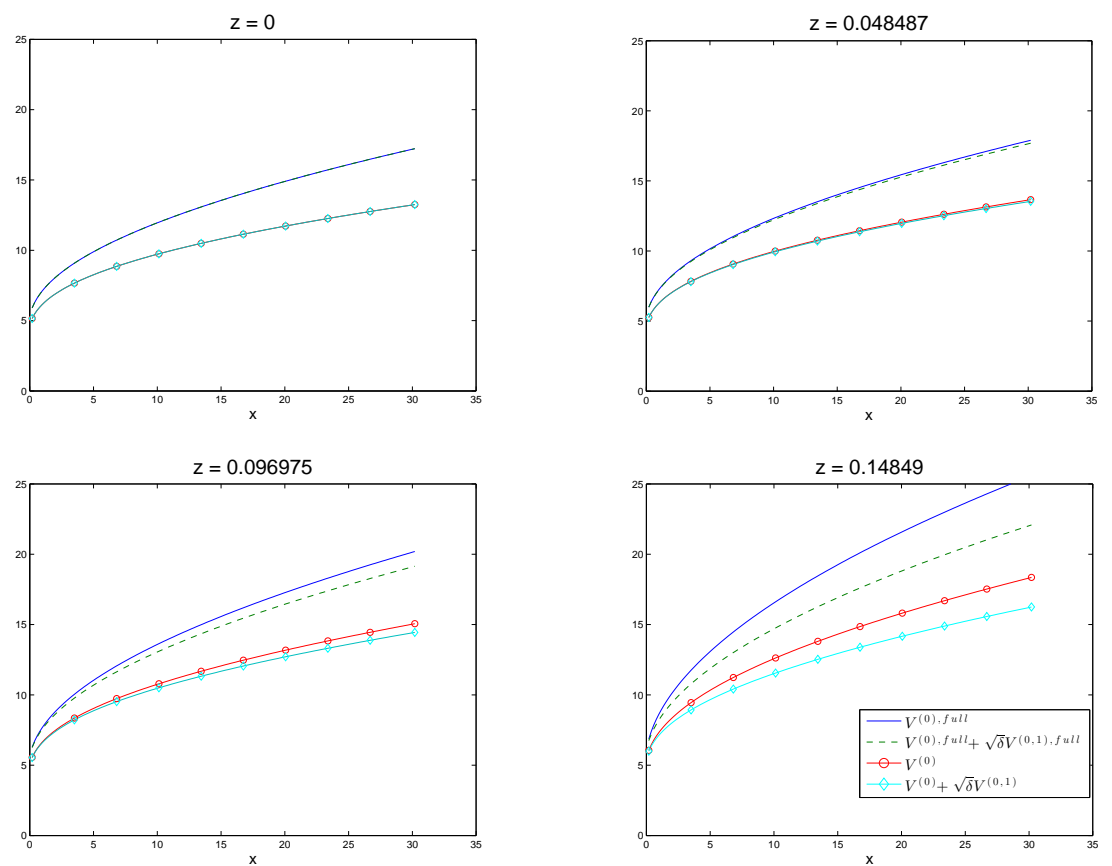

FIG. 6.3. For various levels in $z$, comparisons among the order-zero and order- $\sqrt{\delta}$ terms of the value function expansions, for both full and partial information. The correction for partial information is from the additional risk due to stochasticity in $\widehat{Z}_{t}$, whereas the correction for full information is from the additional risk due to stochasticity in $Z_{t}$.

$\bar{\Sigma}^{\delta}$ is the root to the right-hand side of the Riccati equation in (2.6). Specifically, the root is

$$
\bar{\Sigma}^{\delta} \triangleq-\left(\delta \sigma^{2}+\eta \rho_{2} \sqrt{\delta} \sigma\right)+\sqrt{\left(\delta \sigma^{2}+\eta \rho_{2} \sqrt{\delta} \sigma\right)^{2}+\delta \eta^{2} \sigma^{2}\left(1-\rho_{2}^{2}\right)}
$$

and we have the constant coefficient $b^{\delta}(t)=\bar{b}^{\delta} \triangleq \bar{\Sigma}^{\delta}+\sqrt{\delta} \sigma \eta \rho_{2}=\sqrt{\delta} \sigma \eta \rho_{2}+o(\sqrt{\delta})$ for all $t \in[0, T]$. Also to make the computations easier, we make the change of variables $\tilde{p}=\log p$. Then the PDE for $V^{(0), \delta}$ is equation (4.6) with a logarithmic change-of-variable and homogeneous coefficients:

$$
\left(\frac{\partial}{\partial t}+\frac{\left(\bar{b}^{\delta}\right)^{2}}{2} \frac{\partial^{2}}{\partial z^{2}}+\frac{\left(\frac{1}{2} \rho_{1}^{2} \beta^{2}+z^{2}\right)}{2 \sigma^{2}} \frac{\partial^{2}}{\partial \tilde{p}^{2}}-\frac{z \bar{b}^{\delta}}{\sigma} \frac{\partial^{2}}{\partial z \partial \tilde{p}}-\delta z \frac{\partial}{\partial z}-\frac{\left(\frac{1}{2} \rho_{1}^{2} \beta^{2}+z^{2}\right)}{2 \sigma^{2}} \frac{\partial}{\partial \tilde{p}}\right) \hat{V}^{(0), \delta}=0
$$

with terminal conditions $\left.\hat{V}^{(0), \delta}\right|_{t=T}=\hat{U}$, and where the term $\left\langle y^{2}\right\rangle$ from (4.6) has been written explicitly as $\frac{1}{2} \rho_{1}^{2} \beta^{2}$. Finally, by taking $\gamma_{2}=\frac{1}{2} \gamma_{1}$, we have an easy computation for the utility function's Legendre transform,

$$
\hat{U}(p)=\frac{c_{1}}{1-\gamma_{1}}\left(x^{*}(p)\right)^{1-\gamma_{1}}+\frac{c_{2}}{1-\gamma_{2}}\left(x^{*}(p)\right)^{1-\gamma_{2}}-p x^{*}(p),
$$


where $x^{*}(p)=\left(\frac{\sqrt{c_{2}^{2}+4 c_{1} p}-c_{2}}{2 c_{1}}\right)^{-1 / \gamma_{2}}$ is the root such that $\left.U^{\prime}(x)\right|_{x=x^{*}(p)}=p$.
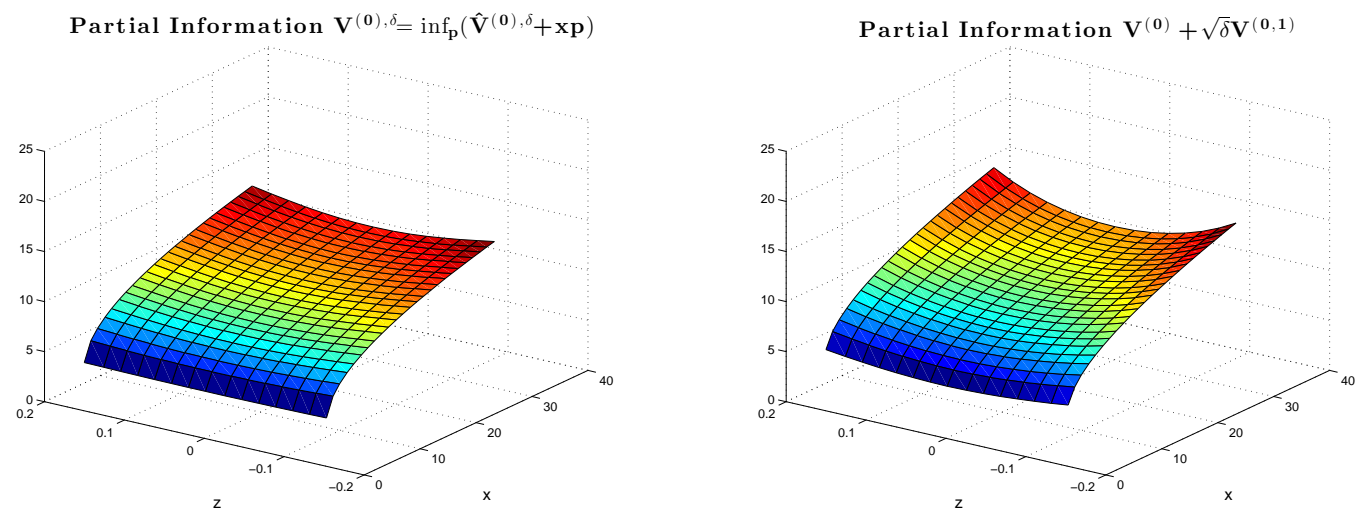

FIG. 6.4. Left: The numerically-inverted $V^{(0), \delta}=\inf _{p}\left(\hat{V}^{(0), \delta}+x p\right)$ where $\hat{V}^{(0), \delta}$ solves equation (6.6), where it is assumed that there is order- $\sqrt{\delta}$ noise in the experts' opinion. Right: The partialinformation approximation $V^{(0)}+\sqrt{\delta} V^{(0,1)}$ approximating solutions to equation (3.7), with the assumption of zero noise in the experts' opinion.

After computing a numerical solution to (6.6), we numerically invert the Legendre transform to obtain a numerical solution to the nonlinear HJB at gridpoints $\left(x_{i}, z_{j}\right)$ with indices $i$ and $j$,

$$
V_{t}^{i, j}=\min _{\ell}\left(\hat{V}_{t}^{\ell, j}+x_{i} e^{\tilde{p}_{\ell}}\right)
$$

where $\hat{V}_{t}^{\ell, j}$ is a numerical solution of (6.6) at gridpoints $\left(\tilde{p}_{\ell}, z_{j}\right)$ with indices $\ell$ and $j$. Figure 6.4 shows a side-by-side comparison of $V^{(0), \delta}$ with the expansion from Figure 6.1 that was computed assuming zero noise in the experts' opinion. Notice how the the solution with no expert noise is higher; this is evidence illustrating that noise in the expert opinions needs to be of order smaller than order $\sqrt{\delta}$, otherwise the value to the investor is the same as if he/she used filtering without expert help.

7. Summary. This paper has shown how fast and slow time scales are useful in analyzing an investment problem with partial information and expert opinions. The methodology is novel because linearity of the PDE associated with convex dual allows for straightforward computation of singular-regular expansions. The main results are simple formulas for a base-term , an order$\sqrt{\varepsilon}$, and an order $-\sqrt{\delta}$ term in the expansion of the optimal value function. Using the Hörmander theorem we were able to show smoothness of solutions to the dual PDE, from which we can verify that the inverse of the dual is optimal and the solution to the nonlinear HJB equation.

The second half of the paper provided numerical simulations focusing on the small- $\delta$ asymptotics. These numerics showed a premium due to partial information, and also demonstrated the importance of having expert opinions with sufficiently low noise. In particular, expert opinions need to be sufficiently confident so as to reduce the initial variance on expected returns to a level that is significantly lower than the perturbations that will occur over the investment period. If expert opinions are too noisy, then investors do not benefit from the extra information. 
Future directions in which to continue the work from this paper include further analysis of the practical strategy, and extension to more specific examples with consumption, multiple assets, and nonlinearity in the filtering equations.

\section{Appendix A. Proofs for Filter Asymptotics.}

Proof. [Proposition 2.1] We have $\mathbb{E}\left(Y_{t}-\widehat{Y}_{t}\right)^{2}=\mathbb{E} Y_{t}^{2}-\mathbb{E} \widehat{Y}_{t}^{2}$, and

$$
\begin{aligned}
& \mathbb{E} Y_{t}^{2}=\mathbb{E}\left(e^{-t / \varepsilon} Y_{0}+\frac{\beta}{\sqrt{\varepsilon}} \int_{0}^{t} e^{-(t-u) / \varepsilon} d B_{u}^{1}\right)^{2} \rightarrow \frac{\beta^{2}}{2} \\
& \mathbb{E} \widehat{Y}_{t}^{2}=\mathbb{E}\left(e^{-t / \varepsilon} \widehat{Y}_{0}+\frac{1}{\sigma^{2}} \int_{0}^{t} e^{-(t-u) / \varepsilon}\left(\Sigma_{11}^{\varepsilon, \delta}(u)+\Sigma_{21}^{\varepsilon, \delta}(u)+\frac{\rho_{1} \sigma \beta}{\sqrt{\varepsilon}}\right) d \nu_{u}\right)^{2} \rightarrow \frac{\rho_{1}^{2} \beta^{2}}{2}
\end{aligned}
$$

as $\varepsilon \rightarrow 0$, so that $\Sigma_{11}^{(0)}(t)=\frac{\beta^{2}\left(1-\rho_{1}^{2}\right)}{2}$ for all $t>0$.

For the cross term $\mathbb{E}\left(Y_{t}-\widehat{Y}_{t}\right)\left(Z_{t}-\widehat{Z}_{t}\right)=\mathbb{E} Y_{t} Z_{t}-\mathbb{E} \widehat{Y}_{t} \widehat{Z}_{t}$, we have

$$
\begin{aligned}
\mathbb{E} Y_{t} Z_{t}= & \mathbb{E}\left(e^{-t\left(\varepsilon^{-1}+\delta\right)} Y_{0} Z_{0}+\frac{\beta}{\sqrt{\varepsilon}} \int_{0}^{t} e^{-(t-u)\left(\varepsilon^{-1}+\delta\right)} Z_{u} d B_{u}^{1}\right. \\
& \left.+\eta \sqrt{\delta} \int_{0}^{t} e^{-(t-u)\left(\varepsilon^{-1}+\delta\right)} Y_{u} d B_{u}^{2}+\sqrt{\frac{\delta}{\varepsilon}} \beta \eta \rho_{12} \int_{0}^{t} e^{-(t-u)\left(\varepsilon^{-1}+\delta\right)} d u\right) \\
\rightarrow 0 \quad \text { as } \varepsilon \rightarrow 0, &
\end{aligned}
$$

and

$$
\begin{aligned}
\mathbb{E} \widehat{Y}_{t} \widehat{Z}_{t}=\mathbb{E}\left\{e^{-t\left(\varepsilon^{-1}+\delta\right)} \widehat{Y}_{0} \widehat{Z}_{0}+\frac{1}{\sigma^{2}} \int_{0}^{t} e^{-(t-u)\left(\varepsilon^{-1}+\delta\right)}\left(\Sigma_{11}^{\varepsilon, \delta}(u)+\Sigma_{21}^{\varepsilon, \delta}(u)+\frac{\rho_{1} \sigma \beta}{\sqrt{\varepsilon}}\right) \widehat{Z}_{u} d \nu_{u}\right. \\
+e^{-t\left(\varepsilon^{-1}+\delta\right)} \widehat{Y}_{0} \widehat{Z}_{0}+\frac{1}{\sigma^{2}} \int_{0}^{t} e^{-(t-u)\left(\varepsilon^{-1}+\delta\right)}\left(\Sigma_{21}^{\varepsilon, \delta}(u)+\Sigma_{22}^{\varepsilon, \delta}(u)+\rho_{2} \sigma \eta \sqrt{\delta}\right) \widehat{Y}_{u} d \nu_{u} \\
+\frac{1}{\sigma^{2}} \int_{0}^{t} e^{-(t-u)\left(\varepsilon^{-1}+\delta\right)}\left(\Sigma_{21}^{\varepsilon, \delta}(u)+\Sigma_{22}^{\varepsilon, \delta}(u)+\rho_{2} \sigma \eta \sqrt{\delta}\right) \\
\left.\times\left(\Sigma_{11}^{\varepsilon, \delta}(u)+\Sigma_{21}^{\varepsilon, \delta}(u)+\frac{\rho_{1} \sigma \beta}{\sqrt{\varepsilon}}\right) d u\right\} \rightarrow 0
\end{aligned}
$$

as $\varepsilon \rightarrow 0$, and hence, $\Sigma_{12}^{(0)}(t)=\Sigma_{21}^{(0)}(t)=0$ for all $t>0$. Finally, for the slow state, we have $\Sigma_{22}^{\varepsilon, \delta}(t)=\mathbb{E}\left(Z_{t}-\widehat{Z}_{t}\right)^{2}=\mathbb{E} Z_{t}^{2}-\mathbb{E} \widehat{Z}_{t}^{2}$ with

$$
\begin{aligned}
\mathbb{E} Z_{t}^{2}= & \mathbb{E}\left(e^{-\delta t} Z_{0}+\sqrt{\delta} \eta \int_{0}^{t} e^{-\delta(t-u)} d B_{u}^{2}\right)^{2}=e^{-2 \delta t} \mathbb{E} Z_{0}^{2}+\delta \eta^{2} \int_{0}^{t} e^{-2 \delta(t-u)} d u \\
\mathbb{E} \widehat{Z}_{t}^{2}= & \mathbb{E}\left(e^{-\delta t} \widehat{Z}_{0}+\frac{1}{\sigma^{2}} \int_{0}^{t} e^{-\delta(t-u)}\left(\Sigma_{21}^{\varepsilon, \delta}(u)+\Sigma_{22}^{\varepsilon, \delta}(u)+\rho_{2} \sigma \eta \sqrt{\delta}\right) d \nu_{u}\right)^{2} \\
= & e^{-2 \delta t} \widehat{Z}_{0}^{2}+\frac{1}{\sigma^{2}} \int_{0}^{t} e^{-2 \delta(t-u)}\left(\Sigma_{21}^{\varepsilon, \delta}(u)+\Sigma_{22}^{\varepsilon, \delta}(u)+\rho_{2} \sigma \eta \sqrt{\delta}\right)^{2} d u \\
& +\frac{1}{\sigma^{2}} \int_{0}^{t} e^{-2 \delta(t-u)}\left(2 \Sigma_{21}^{\varepsilon, \delta}(t)\left(\Sigma_{22}^{\varepsilon, \delta}(u)+\rho_{2} \sigma \eta \sqrt{\delta}\right)+\left(\Sigma_{21}^{\varepsilon, \delta}(t)\right)^{2}\right) d u .
\end{aligned}
$$


Combing these two 2nd moments, we arrive at

$$
\begin{aligned}
\Sigma_{22}^{\varepsilon, \delta}(t)-e^{-2 \delta t} \Sigma_{22}^{\varepsilon, \delta}(0)-\frac{1}{\sigma^{2}} \int_{0}^{t} e^{-2 \delta(t-u)}\left(\delta \sigma^{2} \eta^{2}-\left(\Sigma_{22}^{\varepsilon, \delta}(u)+\rho_{2} \sigma \eta \sqrt{\delta}\right)^{2}\right) d u \\
=-\frac{1}{\sigma^{2}} \int_{0}^{t} e^{-2 \delta(t-u)}\left(2 \Sigma_{21}^{\varepsilon, \delta}(t)\left(\Sigma_{22}^{\varepsilon, \delta}(u)+\rho_{2} \sigma \eta \sqrt{\delta}\right)+\left(\Sigma_{21}^{\varepsilon, \delta}(t)\right)^{2}\right) d u
\end{aligned}
$$

for which the right-hand side goes to zero as $\varepsilon \rightarrow 0$. Hence, $\lim _{\varepsilon} \Sigma_{22}^{\varepsilon, \delta}(t)=\Sigma_{22}^{(0), \delta}(t)$ such that

$$
\Sigma_{22}^{(0), \delta}(t)-e^{-2 \delta t} \Sigma_{22}^{(0), \delta}(0)-\frac{1}{\sigma^{2}} \int_{0}^{t} e^{-2 \delta(t-u)}\left(\delta \sigma^{2} \eta^{2}-\left(\Sigma_{22}^{(0), \delta}(u)+\rho_{2} \sigma \eta \sqrt{\delta}\right)^{2}\right) d u=0
$$

which is the same as the Riccati equation in equation (2.6). This complete the proof of the proposition.

Proof. [Proposition 2.2] We write

$$
\Sigma^{\varepsilon, \delta}(t)=\Sigma^{(0), \delta}(t)+\sqrt{\varepsilon} \widetilde{\Sigma}^{(0), \delta}(t)+\ldots,
$$

where the $\sqrt{\varepsilon}$ correction terms is computed by looking at the limit of $\frac{1}{\sqrt{\varepsilon}}\left(\Sigma^{\varepsilon, \delta}(t)-\Sigma^{(0), \delta}(t)\right)$ as $\varepsilon \rightarrow 0$. Computation of this limit is done using the forms for the variance/covariances that were used in the proof of Proposition 2.1. In particular, all of the terms are zero, except for the following:

$$
\begin{array}{lr}
\frac{1}{\sqrt{\varepsilon}}\left(\mathbb{E} \widehat{Y}_{t}^{2}-\frac{\rho_{1}^{2} \beta^{2}}{2}\right) \rightarrow \frac{\rho_{1} \beta}{\sigma} \Sigma_{11}^{(0), \delta} & \text { as } \varepsilon \rightarrow 0 \\
\frac{1}{\sqrt{\varepsilon}} \mathbb{E} \widehat{Y}_{t} \widehat{Z}_{t} \rightarrow \frac{\rho_{1} \beta}{\sigma}\left(\Sigma_{22}^{(0), \delta}(t)+\rho_{2} \eta \sqrt{\delta}\right) & \text { as } \varepsilon \rightarrow 0 .
\end{array}
$$

This verifies the correction matrix given by the statement of the proposition is in fact the order- $\sqrt{\varepsilon}$ correction in the expansion of $\Sigma^{\varepsilon, \delta}$.

\section{Appendix B. Properties of PDE Solutions.}

B.1. Existence of Classical Solutions to the Dual PDE. We consider log variables, $\tilde{p} \triangleq \log p$ and write equation (3.10) as the following degenerate linear PDE:

$$
\frac{1}{2} A_{1}^{2} \hat{V}^{\varepsilon, \delta}-A_{0} \hat{V}^{\varepsilon, \delta}=0
$$

with terminal condition $\left.\hat{V}^{\varepsilon, \delta}\right|_{t=T}=\hat{U}$, and where

$$
\begin{aligned}
& A_{0} \triangleq-\frac{\partial}{\partial t}+\frac{y}{\varepsilon} \frac{\partial}{\partial y}+\delta z \frac{\partial}{\partial z}+\left(\frac{(\theta+y+z)^{2}}{2 \sigma^{2}}-a^{\varepsilon, \delta}(t)-b^{\varepsilon, \delta}(t)\right) \frac{\partial}{\partial \tilde{p}} \\
& A_{1} \triangleq\left(a^{\varepsilon, \delta}(t) \frac{\partial}{\partial y}+b^{\varepsilon, \delta}(t) \frac{\partial}{\partial z}-\frac{(\theta+y+z)}{\sigma} \frac{\partial}{\partial \tilde{p}}\right) .
\end{aligned}
$$

The vector fields $A_{0}$ and $A_{1}$ are both smooth and bounded at all $(t, \tilde{p}, y, z)$ in compact subsets of $\mathbb{R}^{4}$. Hence, we can verify that $\hat{V}$ has a smooth solution if we check the Hörmander condition (see [CM02, Hör67]). 
B.1.1. The Non-Stationary Case. Consider the expansion from Proposition 2.2 and suppose that $\Sigma_{22}^{(0), \delta}(0)$ is not the positive root to the right-hand side of equation (2.6), then the filter is in a non-stationary state because $\frac{d}{d t} \Sigma_{22}^{\varepsilon, \delta}(t) \neq 0$ for all $t \in[0, T]$. Moreover, $\frac{d}{d t} a^{\varepsilon, \delta}(t)=$ $\sqrt{\varepsilon} \frac{\rho_{1} \beta}{\sigma^{2}} \frac{d}{d t} \Sigma_{22}^{(0), \delta}(t)+o(\sqrt{\varepsilon}) \neq 0$ for all $\delta>0$.

Letting $[\cdot, \cdot]$ denote Lie brackets, we have

$$
\begin{gathered}
{\left[A_{0}, A_{1}\right]=0 \frac{\partial}{\partial t}+\left(\frac{1}{\varepsilon}+\frac{d}{d t}\right) a^{\varepsilon, \delta}(t) \frac{\partial}{\partial y}+\left(\delta+\frac{d}{d t}\right) b^{\varepsilon, \delta}(t) \frac{\partial}{\partial z}} \\
+\left(\frac{y}{\varepsilon \sigma}+\frac{\delta z}{\sigma}+\frac{\theta+y+z}{\sigma^{2}}\left(a^{\varepsilon, \delta}(t)+b^{\varepsilon, \delta}(t)\right)\right) \frac{\partial}{\partial \tilde{p}}, \\
{\left[A_{0},\left[A_{0}, A_{1}\right]\right]=0 \frac{\partial}{\partial t}+\left(\frac{1}{\varepsilon}+\frac{d}{d t}\right)^{2} a^{\varepsilon, \delta}(t) \frac{\partial}{\partial y}+\left(\delta+\frac{d}{d t}\right)^{2} b^{\varepsilon, \delta}(t) \frac{\partial}{\partial z}} \\
-\left\{\frac{y}{\varepsilon^{2} \sigma}+\frac{\delta^{2} z}{\sigma}+\left(\frac{y b^{\varepsilon, \delta}(t)-a^{\varepsilon, \delta}(t)(\theta+z)}{\varepsilon \sigma^{2}}+\frac{\delta z a^{\varepsilon, \delta}(t)-b^{\varepsilon, \delta}(t)(\theta+y)}{\sigma^{2}}\right)\right. \\
{\left[A_{1},\left[A_{0},\left[A_{0}, A_{1}\right]\right]\right]=-\left\{\left(\frac{2}{\varepsilon \sigma} \frac{d}{d t}+\frac{1}{\sigma} \frac{d^{2}}{d t^{2}}\right) a^{\varepsilon, \delta}(t)+\left(\frac{2 \delta}{\sigma} \frac{d}{d t}+\frac{1}{\sigma} \frac{d^{2}}{d t^{2}}\right) b^{\varepsilon, \delta}(t)\right.} \\
\left.+\frac{2}{\sigma^{2}}\left(a^{\varepsilon, \delta}(t)+b^{\varepsilon, \delta}(t)\right) \frac{d}{d t}\left(a^{\varepsilon, \delta}(t)+b^{\varepsilon, \delta}(t)\right)\right\} \frac{\partial}{\partial \tilde{p}}
\end{gathered}
$$

Condition B.1. The Hörmander condition is satisfied if

$$
\operatorname{span}\left\{A_{0},\left[A_{0}, A_{1}\right],\left[A_{0},\left[A_{0}, A_{1}\right]\right],\left[A_{1},\left[A_{0},\left[A_{0}, A_{1}\right]\right]\right]\right\}=\mathbb{R}^{4}
$$

for all $(t, y, z) \in[0, T] \times \mathbb{R} \times \mathbb{R}$, for which the following two conditions are sufficient:

i). vectors $\left[A_{0}, A_{1}\right]$ and $\left[A_{0},\left[A_{0}, A_{1}\right]\right]$ have $\frac{\partial}{\partial y}$ and $\frac{\partial}{\partial z}$ terms that span $\mathbb{R}^{2}$, which happens if

$$
\frac{\left(\frac{1}{\varepsilon}+\frac{d}{d t}\right)^{2} a^{\varepsilon, \delta}(t)}{\left(\frac{1}{\varepsilon}+\frac{d}{d t}\right) a^{\varepsilon, \delta}(t)} \neq \frac{\left(\delta+\frac{d}{d t}\right)^{2} b^{\varepsilon, \delta}(t)}{\left(\delta+\frac{d}{d t}\right) b^{\varepsilon, \delta}(t)}
$$

ii). and the dimension in $\frac{\partial}{\partial \tilde{p}}$ is spanned by $\left[A_{1},\left[A_{0},\left[A_{0}, A_{1}\right]\right]\right]$, which is the case if

$$
\begin{aligned}
\left(\frac{2}{\varepsilon \sigma} \frac{d}{d t}+\frac{1}{\sigma} \frac{d^{2}}{d t^{2}}\right) a^{\varepsilon, \delta}(t)+ & \left(\frac{2 \delta}{\sigma} \frac{d}{d t}+\frac{1}{\sigma} \frac{d^{2}}{d t^{2}}\right) b^{\varepsilon, \delta}(t) \\
& +\frac{2}{\sigma^{2}}\left(a^{\varepsilon, \delta}(t)+b^{\varepsilon, \delta}(t)\right) \frac{d}{d t}\left(a^{\varepsilon, \delta}(t)+b^{\varepsilon, \delta}(t)\right) \\
\neq 0 . &
\end{aligned}
$$

For any $t \in(0, T]$, from the expansion in Proposition 2.2 we have $a^{\varepsilon, \delta}(t) \sim \frac{\rho_{1} \beta}{\sqrt{\varepsilon}}, \frac{d}{d t} a^{\varepsilon, \delta}(t) \sim$ $\sqrt{\varepsilon} \frac{\rho_{1} \beta}{\sigma^{2}} \frac{d}{d t} \Sigma_{22}^{(0), \delta}(t)$ and $\frac{d^{2}}{d t^{2}} a^{\varepsilon, \delta}(t) \sim \sqrt{\varepsilon} \frac{\rho_{1} \beta}{\sigma^{2}} \frac{d^{2}}{d t^{2}} \Sigma_{22}^{(0), \delta}(t)$. Using the expansion, we see that Part (i) of 
the Condition B.1 is satisfied if

$$
\frac{\left(\frac{1}{\varepsilon}+\frac{d}{d t}\right)^{2} a^{\varepsilon, \delta}(t)}{\left(\frac{1}{\varepsilon}+\frac{d}{d t}\right) a^{\varepsilon, \delta}(t)} \sim \frac{1}{\varepsilon} \gg \frac{\left(\delta+\frac{d}{d t}\right)^{2} b^{\varepsilon, \delta}(t)}{\left(\delta+\frac{d}{d t}\right) b^{\varepsilon, \delta}(t)},
$$

which is the case when $\varepsilon \ll 1$ and $\rho_{1} \neq 0$. Part (ii) of the Condition B.1 is checked by looking at terms of order $\varepsilon^{-1}$, and is satisfied for $\varepsilon \ll 1 \rho_{1} \neq 0$ if

$$
\frac{d}{d t} a^{\varepsilon, \delta}(t) \neq 0 \quad \forall t \in[0, T],
$$

which is the case if $\frac{d}{d t} \Sigma_{22}^{\varepsilon, \delta}(t) \neq 0$. This verifies the Hörmander condition, and we can conclude that equation (B.1) has smooth classical solutions.

B.1.2. The Stationary Case. If $\Sigma^{\varepsilon, \delta}(0)$ is such that it solves the matrix Riccati equation on the right-hand side of (2.5), then $\frac{d}{d t} a^{\varepsilon, \delta}(t)=0=\frac{d}{d t} b^{\varepsilon, \delta}(t)$ for all time, and the coefficients are constant with $a^{\varepsilon, \delta}(t)=\bar{a}^{\varepsilon, \delta}$ and $b^{\varepsilon, \delta}(t)=\bar{b}^{\varepsilon, \delta}$ for all $t \geq 0$ (Section 6.4 considers the $\varepsilon$ limit of this stationary case). Time homogeneity makes verification of the Hörmander condition slightly different from Appendix B.1.

We define the vector fields

$$
\begin{aligned}
& A_{0} \triangleq \frac{y}{\varepsilon} \frac{\partial}{\partial y}+\delta z \frac{\partial}{\partial z}+\left(\frac{(\theta+y+z)^{2}}{2 \sigma^{2}}-\bar{a}^{\varepsilon, \delta}-\bar{b}^{\varepsilon, \delta}\right) \frac{\partial}{\partial \tilde{p}}, \\
& A_{1} \triangleq\left(\bar{a}^{\varepsilon, \delta} \frac{\partial}{\partial y}+\bar{b}^{\varepsilon, \delta} \frac{\partial}{\partial z}-\frac{(\theta+y+z)}{\sigma} \frac{\partial}{\partial \tilde{p}}\right),
\end{aligned}
$$

so that the PDE is $\left(\frac{\partial}{\partial t}+\frac{1}{2} A_{1}^{2}-A_{0}\right) \hat{V}^{\varepsilon, \delta}=0$. Then the Lie brackets are

$$
\begin{aligned}
& {\left[A_{0}, A_{1}\right]=\frac{\bar{a}^{\varepsilon, \delta}}{\varepsilon} \frac{\partial}{\partial y}+\delta \bar{b}^{\varepsilon, \delta}(t) \frac{\partial}{\partial z}+\left(\frac{y}{\varepsilon \sigma}+\frac{\delta z}{\sigma}+\frac{\theta+y+z}{\sigma^{2}}\left(\bar{a}^{\varepsilon, \delta}+\bar{b}^{\varepsilon, \delta}\right)\right) \frac{\partial}{\partial \tilde{p}},} \\
& {\left[A_{1},\left[A_{0}, A_{1}\right]\right]=\left(-2\left(\frac{\bar{a}^{\varepsilon, \delta}}{\varepsilon \sigma}+\frac{\delta \bar{b}^{\varepsilon, \delta}}{\sigma}\right)-\frac{\left(\bar{a}^{\varepsilon, \delta}+\bar{b}^{\varepsilon, \delta}\right)^{2}}{\sigma^{2}}\right) \frac{\partial}{\partial \tilde{p}},}
\end{aligned}
$$

from which we see that $\operatorname{span}\left\{A_{1},\left[A_{0}, A_{1}\right],\left[A_{1},\left[A_{0}, A_{1}\right]\right]\right\}=\mathbb{R}^{3}$ if $\frac{1}{\varepsilon} \neq \delta$ and $-2\left(\frac{\bar{a}^{\varepsilon, \delta}}{\varepsilon}+\delta \bar{b}^{\varepsilon, \delta}\right) \neq$ $\frac{1}{\sigma}\left(\bar{a}^{\varepsilon, \delta}+\bar{b}^{\varepsilon, \delta}\right)^{2}$. This verifies the parabolic Hörmander condition.

B.2. Accuracy of HJB Expansion. This appendix will give an idea of how to use the expansion of the dual solution to justify the formal expansion of the solution to the nonlinear HJB; all that is required is regularity. Consider the $\sqrt{\delta}$ expansion of $\hat{V}^{(0), \delta}$ given in equation (5.1), and its inverse Fenchel-Legendre transform,

$$
V^{(0), \delta}(t, x, z)=\inf _{p \geq 0}\left(\hat{V}^{(0), \delta}(t, p, z)+x p\right) .
$$

From first-order conditions we find that $\hat{V}_{p}^{(0), \delta}\left(t, p^{*}(x), z\right)=-x$ at the critical point. Now using the implicit function theorem we obtain the expansion

$$
p^{*}(x)=p^{(0)}(x)+\sqrt{\delta} p^{(1)}(x)+\delta p^{(2)}(x)+\ldots
$$


where $p^{(0)}(x)$ is the critical point such that $V^{(0)}(t, x, z)=\hat{V}^{(0)}\left(t, p^{(0)}(x), z\right)+x p^{(0)}(x)$, where $V^{(0)}$ is the solution to equation (5.5). Now using the $\sqrt{\delta}$ expansions and regularity, we find

$$
\begin{aligned}
V^{(0), \delta}(t, x, z) & =\hat{V}^{(0), \delta}\left(t, p^{*}(x), z\right)+x p^{*}(x) \\
& =\hat{V}^{(0)}\left(t, p^{*}(x), z\right)+\sqrt{\delta} \hat{V}^{(0,1)}\left(t, p^{*}(x), z\right)+x p^{(0)}(x)+\sqrt{\delta} x p^{(1)}(x)+o(\sqrt{\delta}) \\
& =\hat{V}^{(0)}\left(t, p^{(0)}(x), z\right)+\sqrt{\delta} p^{(1)}(x) \hat{V}_{p}^{(0)}\left(t, p^{(0)}(x), z\right)+\sqrt{\delta} \hat{V}^{(0,1)}\left(t, p^{(0)}(x), z\right) \\
& \quad+x p^{(0)}(x)+\sqrt{\delta} x p^{(1)}(x)+o(\sqrt{\delta}) \\
& =\hat{V}^{(0)}\left(t, p^{(0)}(x), z\right)+\sqrt{\delta} \hat{V}^{(0,1)}\left(t, p^{(0)}(x), z\right)+x p^{(0)}(x)+o(\sqrt{\delta}) \\
& =V^{(0)}(t, x, z)+\sqrt{\delta} \hat{V}^{(0,1)}\left(t, p^{(0)}(x), z\right)+o(\sqrt{\delta}) \\
& =V^{(0)}(t, x, z)+\sqrt{\delta} V^{(0,1)}(t, x, z)+o(\sqrt{\delta}),
\end{aligned}
$$

where the last equality follows from equation (5.8) of Proposition 5.3. As mentioned in Remark 1, the above calculation is the beginning of a proof to show $o(\sqrt{\varepsilon})+o(\sqrt{\delta})$ accuracy of the expansion of $V^{\varepsilon, \delta}$ using order $\sqrt{\varepsilon}$ and $\sqrt{\delta}$ terms given by Propositions 5.5 and 5.3, respectively.

\section{REFERENCES}

[BC09] A. Bain and D. Crisan. Fundamentals of Stochastic Filtering. Springer, New York, NY, 2009.

[BEW88] T. Bollerslev, R. F. Engle, and J. M. Wooldridge. A capital asset pricing model with time-varying covariances. Journal of Political Economy, 96(1):116-31, February 1988.

[BL91] F. Black and R. Litterman. Asset allocation combining investor views with market equilibrium. Journal of Fixed Income, 1(2):7-18, September 1991.

[BL92] F. Black and R. Litterman. Global portfolio optimization. Financial Analysts Journal, 48(5):28-43, Sep.-Oct. 1992.

[BR05] N. Bäuerle and U. Rieder. Portfolio optimization with unobservable Markov-modulated drift process. Journal of Applied Probability, 42(2):362-378, 2005.

[Bre98] M. J. Brennan. The role of learning in dynamic portfolio decisions. European Finance Review, 1(3):295306, 1998.

[Bre06] S. Brendle. Portfolio selection under incomplete information. Stochastic Processes and their Applications, 116(5):701 - 723, 2006.

[BUV12] N. Bäuerle, S. Urban, and L. Veraart. The relaxed investor with partial information. SIAM Journal on Financial Mathematics, 3(1):304-327, 2012.

[Car09] R. Carmona. From Markovian to partially observable models. In Indifference Pricing. Princeton University Press, 2009.

[CM02] P. Cattiaux and L. Mesnager. Hypoelliptic non-homogeneous diffusions. Probability Theory and Related Fields, 123(4):453-483, August 2002.

[CV99] J. Y. Campbell and L. M. Viceira. Consumption and portfolio decisions when expected returns are time varying. The Quarterly Journal of Economics, 114(2):433-495, May 1999.

[ESB10] R.J. Elliott, T.K. Siu, and A. Badescu. On mean-variance portfolio selection under a hidden Markovian regime-switching model. Economic Modeling, 27(3):678-686, 2010.

[FGW12] R. Frey, A. Gabih, and R. Wunderlich. Portfolio optimization under partial information with expert opinions. International Journal of Theoretical and Applied Finance, 15(1):1-18, February 2012.

[FPS14] J.-P. Fouque, A. Papanicolaou, and R. Sircar. Filtering and portfolio optimization with stochastic unobserved drift in asset returns. Communications in Mathematical Sciences, 2014. To appear.

[FPSS11] J.-P. Fouque, G. Papanicolaou, R. Sircar, and K. Solna. Multiscale Stochastic Volatility for Equity, Interest Rate, and Credit Derivatives. Cambridge University Press, 2011.

[FSZ13] J.-P. Fouque, R. Sircar, and T. Zariphopoulou. Portfolio optimization \& stochastic volatility asymptotics. 2013. Preprint. 
[HL88] C. Huang and R. Litzenberger. Foundations of Financial Economics. Prentice Hall, Englewood Cliffs, NJ, 1988.

[Hör67] L. Hörmander. Hypoelliptic second order differential equations. Acta Math, 117:147-171, 1967.

[JS02a] M. Jonsson and R. Sircar. Optimal investment problems and volatility homogenization approximations. In A. Bourlioux, M. Gander, and G. Sabidussi, editors, Modern Methods in Scientific Computing and Applications, volume 75 of NATO Science Series II, pages 255-281. Kluwer, 2002.

[JS02b] M. Jonsson and R. Sircar. Partial hedging in a stochastic volatility environment. Mathematical Finance, 12(4):375-409, October 2002.

[Kal80] G. Kallianpur. Stochastic Filtering Theory. Springer-Verlag, New York, NY, 1980.

[KO96] T. S. Kim and E. Omberg. Dynamic nonmyopic portfolio behavior. Review of Financial Studies, 9(1):141$161,1996$.

[KS98] I. Karatzas and S. Shreve. Methods of Mathematical Finance. Springer-Verlag, 1998.

[KS99] D. Kramkov and W. Schachermayer. The asymptotic elasticity of utility functions and optimal investment in incomplete markets. The Annals of Applied Probability, 9(3):904-950, 1999.

[Mar52] H. M. Markowitz. Portfolio selection. The Journal of Finance, 7(1):77-91, March 1952.

[Mer69] R. C. Merton. Lifetime portfolio selection under uncertainty: the continous-time case. Rev. Econom. Statist., 51:247-257, 1969.

[Pap13] A. Papanicolaou. Dimension reduction in discrete time portfolio optimization with partial information. SIAM Journal on Financial Mathematics, 4(1):916-960, 2013.

[Pha09] H. Pham. Continuous-time Stochastic Control and Optimization with Financial Applications. Springer, 2009.

[PRS11] J. Park, B. Rozovsky, and R. Sowers. Efficient nonlinear filtering of a singularly perturbed stochastic hybrid system. LMS J. Computational Mathematics, 14:254-270, 2011.

[PS14] A. Papanicolaou and K. Spiliopoulos. Filtering the maximum likelihood for multiscale problems. SIAM J. Multiscale Modeling and Simulation, 12(3):1193-1229, 2014.

[Rog13] L.C.G. Rogers. Optimal Investment. Springer, 2013.

[Sch03] W. Schachermayer. Introduction to the mathematics of financial markets. In Pierre Bernard, editor, Lectures on Probability Theory and Statistics, Saint-Fleur summer school 2000, number 1816 in Lecture Notes in Mathematics, pages 111-177. Springer Verlag, 2003.

[SD14] R. Sircar and Y. Dong. Time-inconsistent portfolio investment problems. In D. Crisan, B. Hambly, and T. Zariphopoulou, editors, Stochastic Analysis and Applications. Springer, 2014. forthcoming.

[SH04] J. Sass and U. Haussmann. Optimizing the terminal wealth under partial information: The drift process as a continuous time Markov chain. Finance and Stochastics, 8(4):553-577, November 2004.

[SWZ10] K. Schöttle, R. Wernér, and R. Zagst. Comparison and robustification of Bayes and Black-Litterman models. Mathematical Methods of Operations Research, 71(3):453-475, June 2010.

[Wac02] J. Wachter. Portfolio and consumption decisions under mean-reverting returns: An exact solution for complete markets. Journal Financial and Quantitative Analysis, 37(1):63-91, 2002.

[YZ13] G. Yin and Q. Zhang. Continuous-Time Markov Chains and Applications: A Two-Time-Scale Approach, volume 37 of Stochastic Modelling and Applied Probability. Springer Verlag, New York, 2013. 